Tesis doctoral. Departament de Medicina Interna. Universitat Autònoma de Barcelona

\title{
Dislipemia diabética
}

\section{Evaluación y tratamiento}

Ana María Wägner Fahlin

Directores:

Antonio Pérez Pérez

Jordi Ordóñez Llanos

Tutor:

Alberto de Leiva 


\section{AGRADECIMIENTOS}

Al Dr Antonio Pérez Pérez, Adjunto de Endocrinología y Nutrición del Hospital Sant Pau, co-director de esta tesis, por su interés, consejos y buenas ideas y por su colaboración en el reclutamiento de pacientes.

Al Prof Jordi Ordóñez, Jefe de la Sección de Lípidos del Servicio de Bioquímica del Hospital Sant Pau, Profesor Titular de Bioquímica y Biología Molecular de la UAB, codirector de esta tesis, por su enseñanza en el orden y el rigor, sin olvidar su colaboración como control sano en el estudio de la lipemia postprandial.

A mi tutor, el Prof. Alberto de Leiva, Director del Servicio de Endocrinología y Nutrición, Catedrático de Medicina de la UAB, por su apoyo y confianza, sin cuya colaboración esta tesis no habría sido posible.

Al Prof. Francesc González Sastre, Director del Servicio de Bioquímica, Catedrático y Director del Dto. de Bioquímica y Biología Molecular de la UAB, por facilitar la realización de la presente tesis.

A Francisco Blanco, Jefe de Sección del Servicio de Bioquímica del Hospital Sant Pau, por su ejemplo en capacidad de superación de momentos difíciles, y por darme la oportunidad de participar en nuevos proyectos.

A Josep Julve, Dr en Biología, investigador en la Sección de Lípidos del Hospital Sant Pau, por su colaboración en la determinación de las actividades de lipoproteinlipasa y lipasa hepática, durante la finalización de su propia tesis.

A Rosa Bonet, bióloga, por su inestimable ayuda y paciencia para enseñarme cómo dar los primeros pasos en el laboratorio, por su colaboración en el procesamiento de muestras, por su orden y método. 
A Rosa Arcelus, farmacéutica, por su colaboración en la determinación del retinilpalmitato, y por su participación en el estudio de lipemia postprandial como control sana.

A Jose Luis Sánchez Quesada, Dr en Biología, investigador en la Sección de Lípidos del Hospital Sant Pau, por su colaboración en la puesta a punto de varios métodos, y su visión crítica de los trabajos.

A Óscar Jorba, biólogo y Esther Alonso, química, por su paciencia y buen humor al introducirme en la electroforesis en gel de poliacrilamida, y por su colaboración en la determinación de tamaño de partícula LDL.

A Laia Bayen, técnica de laboratorio, por su inestimable ayuda en la obtención de datos. A Agustina Castellví, enfermera, por su ayuda en el procesamiento de muestras y a Pepi Gascón, auxiliar, por su optimismo y buen humor contagiosos, y por colaborar como control sana en el estudio de la lipemia postprandial

Al resto de miembros del laboratorio de la Sección de Lípidos, por hacerme sentir como en casa entre ellos.

A Mariano Cortés, por su inestimable ayuda en la utilización de métodos estadísticos.

Al personal de Extracciones, por su colaboración desinteresada en la obtención de muestras, y por hacer a los voluntarios más cómoda su participación en el estudio.

A Pedro Betancor León, Catedrático de Medicina Interna y ex-Decano de la Facultad de Ciencias de la Salud de Las Palmas de Gran Canaria y a Javier Nóvoa Mogollón, profesor titular de Endocrinología de la Universidad de Las Palmas, Jefe de Sección de Endocrinología del Hospital Insular de Las Palmas, por ayudarme en la toma de decisiones a la hora de iniciar mi formación como especialista.

A Mercedes Rigla, por su participación en el reclutamiento de pacientes, por su colaboración en varios de los trabajos, por su espíritu crítico y sus acertados consejos. 
A Rosa Corcoy, Assumpta Caixæ̀s, Susan Webb y demás adjuntos del Servicio de Endocrinología del Hospital de Sant Pau por sus enseñanzas y colaboración en el reclutamiento de pacientes. En especial también a Jose $M^{a}$ - Pou, por su participación como control sano.

A Jaume Puig, por su ejemplo, generosidad y apoyo en el trabajo diario durante toda la Residencia. A Águeda Caballero, por su hospitalidad y acogida en Barcelona.

A Emilio Ortega, Antonio Mestrón y demás residentes por su colaboración en el reclutamiento de pacientes y a Marta Hernández, por su colaboración en la realización de trabajos relacionados.

A Fernando Calvo, coautor del primer trabajo de esta tesis, por su eficiencia, y su espíritu colaborador y entusiasta.

A Esther Martín, Justa Úbeda, Miguel A María, Luisa Erdozain, Emilia Belda y el resto del equipo de enfermería y a Ana Martí, Paquita Cañas, Ana Oviedo y resto del personal administrativo, por su colaboración, tanto programada como espontánea, en la atención a los pacientes y en la solución de problemas "técnicos".

A Carmen Mayoral, Miquel Santaló, Mercedes Puy, Graciela Ferrari, Esteban Guardia, Alex Roca-Cusachs, José Rodríguez Espinosa, Jordi Farrerons, José Rodríguez Maqueda, y Britta Wägner, por su colaboración como controles sanos en el estudio de lipemia postprandial.

A todos los pacientes incluídos en los diversos estudios, por su colaboración desinteresada, sin los cuales no habría sido posible la realización de este trabajo. 
A la ayuda recibida de la Generalitat de Catalunya, mediante la Beca 1999FI 0712 , gracias a la cual he podido dedicar dos años a los trabajos de la presente tesis. A los laboratorios Parke Davis (ahora Pfizer), por su colaboración en el trabajo sobre el tratamiento farmacológico de la dislipemia diabética.

A Jose Luis Porta, por su apoyo incondicional,...e inagotable paciencia.

A Elena y a Vicki Wägner, por las horas robadas, y a Jesús Peco, por estar siempre dispuesto a escuchar y a dar buenos consejos.

Y, finalmente, a Britta y a Lamberto Wägner, mis padres, por proporcionarme herramientas y por estar siempre cerca, incluso en la distancia. 


\section{ÍNDICE}


Agradecimientos: $\quad 2$

$\begin{array}{ll}\text { Índice: } & 6\end{array}$

$\begin{array}{ll}\text { Introducción: } & 11\end{array}$

$\begin{array}{ll}\text {-Dislipemia diabética: } & 12\end{array}$

Componentes clásicos: $\quad 12$

- Colesterol de LDL 12

riesgo CV 12

métodos de medida 13

CLDL y diabetes $\quad 14$

- $\quad$ Colesterol de HDL 15

riesgo CV 15

métodos de medida $\quad 15$

CHDL y diabetes $\quad 16$

- Triglicéridos 17

$\begin{array}{ll}\text { riesgo } \mathrm{CV} & 17\end{array}$

métodos de medida $\quad 18$

triglicéridos y diabetes $\quad 19$

- Resumen 20

Componentes no clásicos: $\quad 20$

- Apolipoproteina B: 20

riesgo $\mathrm{cv} 20$

métodos de medida $\quad 21$ 
apolipoproteina B y diabetes

resumen

- Partículas de LDL pequeñas y densas: 22

riesgo CV

23

métodos de medida

24

LDL pequeñas y densas y diabetes 25

resumen

26

- Lipemia postprandial:

riesgo CV

27

métodos de medida

28

lipemia postprandial y diabetes

31

resumen

-Evaluación y tratamiento de la dislipemia diabética:

Evaluación y objetivos terapéuticos:

Tratamiento de la dislipemia diabética: 38

Medidas higiénico-dietéticas:

Dieta y pérdida ponderal

- Efecto sobre los componentes clásicos 38

- Efecto sobre apolipoproteina B 40

- Efecto sobre el tamaño de las LDL 41

- Efecto sobre la lipemia postprandial 42

Ejercicio físico 43

- Efecto sobre componentes clásicos 43

- Efecto sobre apolipoproteina B 44

- Efecto sobre el tamaño de las LDL $\quad 44$

- Efecto sobre la lipemia postprandial 45

Resumen 46 
Control glucémico $\quad 46$

- $\quad$ Efecto sobre componentes clásicos 46

- $\quad$ Efecto sobre apolipoproteina B 48

- $\quad$ Efecto sobre el tamaño de las LDL 48

- Efecto sobre la lipemia postprandial 49

- Resumen 50

Fármacos hipolipemiantes $\quad 50$

- Efecto sobre el riesgo cardiovascular 50

- Efecto sobre componentes clásicos 53

- Efecto sobre apolipoproteina B $\quad 55$

- Efecto sobre el tamaño de las LDL 56

- Efecto sobre la lipemia postprandial $\quad 57$

- Resumen 58

Justificación y objetivos: 59

Artículos derivados de esta tesis: 63

Discusión y resumen de resultados: 64

Evaluación de la dislipemia diabética 65

Optimización de la determinación del cLDL $\quad 65$

Apolipoproteína B 68

Tamaño de la LDL $\quad 71$

Lipemia postprandial $\quad 74$

$\begin{array}{ll}\text { Resumen } & 76\end{array}$

Tratamiento farmacológico de la dislipemia diabética $\quad 77$

Comparación entre un fibrato y una estatina $\quad 78$

Tratamiento combinado $\quad 81$

Resumen 82 
Conclusiones finales:

Bibliografía:

Tablas:

109

Figuras:

Abreviaturas:

Otras publicaciones relacionadas: 


\section{INTRODUCCIÓN}




\section{DISLIPEMIA DIABÉTICA}

El término dislipemia diabética hace referencia a las alteraciones lipídicas características de la diabetes tipo 2, pero no de la diabetes tipo 1. Al contrario de lo que ocurre en la diabetes tipo 1 (DCCT Research Group 1992, Pérez 1997, Caixàs 1997, Pérez 2000, Sánchez-Quesada 1996), las alteraciones presentes en la diabetes tipo 2, aunque mejoran, no desaparecen con la optimización del control glucémico (Caixàs 1997, Taskinen 1992). Entre los componentes de la dislipemia diabética existen unos de fácil medición en cualquier laboratorio clínico, y que la definen clásicamente (hipertrigliceridemia moderada, colesterol de HDL (cHDL) bajo y colesterol de LDL (cLDL) normal o levemente elevado (Taskinen 1996), y otros, que también son típicos de los pacientes con diabetes tipo 2, pero que no se determinan rutinariamente (componentes no clásicos), como el predominio de partículas pequeñas y densas, el aumento de la apolipoproteina $B(a p o B)$ y el aumento de la lipemia postprandial. A continuación revisaremos cada uno de los componentes en relación a su prevalencia, su asociación con la enfermedad cardiovascular y su medición.

\section{COMPONENTES CLÁSICOS}

\section{1-Colesterol de LDL}

\section{1a-Colesterol de LDL y riesgo cardiovascular.}

Las partículas de LDL, contienen aproximadamente el $70 \%$ de todo el colesterol circulante, y su función es transportar el colesterol procedente del hígado a los tejidos periféricos. El poder aterogénico del CLDL está firmemente establecido, y se basa en estudios experimentales, epidemiológicos y de intervención. Los estudios experimentales demuestran que las partículas de LDL pueden atravesar el endotelio, adherirse en la matriz extracelular al colágeno subendotelial (Boren 1998, Camejo 1998), y ser trasformadas y fagocitadas por los macrófagos y células musculares lisas, favoreciendo el desarrollo de la placa de ateroma y la proliferación vascular (Heinecke 1998, Hurt-Camejo 1997). Estudios epidemiológicos longitudinales demuestran que el 
cLDL es un mejor predictor de enfermedad cardiovascular que el colesterol total, y que por cada $30 \mathrm{mg} / \mathrm{dl}(0,8 \mathrm{mmol} / \mathrm{l})$ que aumenta su concentración, el riesgo de padecer un evento coronario se duplica (Assmann 1998). La reducción de eventos coronarios que se obtiene en los estudios de intervención con tratamientos hipocolesterolemiantes es proporcional a la reducción de colesterol, independientemente del fármaco o medida no farmacológica utilizados (Bucher 1999).

En pacientes con diabetes, el cLDL también es el más potente predictor de enfermedad coronaria según el UKPDS (Turner 1998), y mantiene su poder predictor incluso a concentraciones inferiores a $130 \mathrm{mg} / \mathrm{dl}$ (Howard 2000). Su poder aterogénico en el sujeto con diabetes está asimismo avalado por estudios de intervención con tratamientos hipocolesterolemiantes que demuestran una reducción del riesgo cardiovascular (ver más adelante).

\section{1b-Medición del colesterol de LDL}

El método de referencia para la determinación del cLDL es la ultracentrifugación secuencial (Havel 1955), pero por su escasa practicabilidad se utiliza un método designado de comparación: la betacuantificación. Ésta consiste en la separación de las partículas de VLDL (densidad < 1,006 Kg/l) por flotación tras ultracentrifugación. Posteriormente, en el infranadante de esta ultracentrifugación, se precipitan las partículas con apolipoproteina B (apoB) (IDL, LDL y lipoproteína (a) [lp(a)]). Tras someter la mezcla a una centrifugación a baja velocidad, se mide el colesterol total del sobrenadante, que contiene el colesterol de las partículas de alta densidad (cHDL) (ver Figura 1). La concentración de cLDL será el resultado de restar dicho cHDL del colesterol total del infranadante (antes de la precipitación), e incluirá no sólo el cLDL, sino también el colesterol de las partículas de densidad intermedia (cIDL) y el de la Ip(a) (Bachorik 1997). Dada la complejidad de este método, se han propuesto alternativas más sencillas, asequibles a la práctica clínica para la estimación del CLDL. La fórmula de Friedewald $(\mathrm{cLDL}=$ colesterol total $-\mathrm{cHDL}$ - [triglicéridos $(\mathrm{en} \mathrm{mg} / \mathrm{dl}) / 5$ ó triglicéridos (en $\mathrm{mmol} / \mathrm{l} / 2,17]$ ), que es la más utilizada, asume que la razón 
colesterol/triglicéridos de las partículas de VLDL es constante (Friedewald 1972), premisa que no es válida en presencia de quilomicronemia o aumento del número partículas VLDL o IDL (Friedewald 1972, Bachorik 1995, Sentí 1991). Por ello, se han desarrollado fórmulas alternativas (Planella 1997, De Long 1986, Rao 1988) y métodos de medición directa del cLDL (McNamara 1995, Okabe 1999, Nauck 2000, Nauck 2000a, Esteban 2000), pero hasta el momento ninguno ha sido aceptado de forma generalizada.

\section{1c-Colesterol de LDL y diabetes}

La concentración de cLDL en pacientes con diabetes mellitus tipo 2 no suele diferir en gran medida de la que se encuentra en sujetos sin diabetes, tanto en hombres como en mujeres (ver tablas 1a y 1 b). El estudio de Framingham (que incluye a un $4 \%$ de pacientes con diabetes tipo 1) muestra concentraciones de cLDL similares en las mujeres (134 vs $132 \mathrm{mg} / \mathrm{dl}$ ), pero más bajas en los hombres diabéticos respecto a sus controles (132 vs $137 \mathrm{mg} / \mathrm{dl}, \mathrm{p}<0,05)$, tras ajustar por edad (Siegel 1996). Sin embargo, los datos procedentes del United Kingdom Prospective Diabetes Study (UKPDS) muestran un cLDL superior en mujeres con diabetes que sin diabetes (150,5 vs $133,9 \mathrm{mg} / \mathrm{dl}$ ), y similar en hombres con y sin diabetes (UK Prospective Study Group 1994, UK Prospective Study Group 1997). El estudio de San Antonio muestra, en los sujetos con diabetes no hispanos, concentraciones de cLDL que son equiparables a las obtenidas en los estudios antes mencionados (Haffner 1998)(ver tablas 1a y 1b). Por otro lado, en los estudios de intervención que proporcionan datos sobre los pacientes con diabetes, las concentraciones de cLDL son también similares o más bajas en dicho subgrupo que en los sujetos sin diabetes, aunque hay que tener en cuenta que se trata de poblaciones seleccionadas por criterios de inclusión (Koskinen 1992, Goldberg 1998, Pyörälä 1998, Hoogwerf 1999) (ver tabla 2).

La prevalencia de hipercolesterolemia (cLDL > $160 \mathrm{mg} / \mathrm{dl}$ ) según el Framingham Offspring Study es del $22,4 \%$ y $35 \%$ en hombres y mujeres con diabetes, respectivamente, porcentajes que son similares a la población no diabética de la 
misma edad (27\% y $22,2 \%$, respectivamente) (Siegel 1996). En el UKPDS, por otro lado, solamente un $8 \%$ de los sujetos con diabetes tipo 2 mostraron concentraciones de cLDL por encima del percentil 97,5 de la población no diabética (UK Prospective Study Group 1994).

\section{2-Colesterol de HDL}

\section{2a-Colesterol de HDL y riesgo cardiovascular}

Las partículas de HDL son protagonistas del transporte reverso de colesterol (Pieters 1994), pero además tienen propiedades antioxidantes (Parthasarathy 1990, Mackness 1993) y reparadoras del endotelio (Pomeranz 1985), y se les atribuye un efecto antiinflamatorio sobre el proceso de aterosclerosis (Cockerill 1995, Vadas 1997). Numerosos estudios epidemiológicos han establecido que la concentración de cHDL es un factor predictivo independiente de la incidencia de enfermedad coronaria (Miller 1975, Castelli 1986, Gordon 1986, Whatkins 1986, Bainton 1992, Assmann 1996). Se estima que por cada $\mathrm{mg} / \mathrm{dl}$ ( 0,03 $\mathrm{mmol} / \mathrm{l})$ que aumenta el $\mathrm{cHDL}$, disminuye el riesgo de enfermedad coronaria en un 2-3\% (Gordon 1989). Los estudios de intervención también demuestran una reducción de la incidencia de infarto agudo de miocardio y de enfermedad cerebrovascular tras la disminución de triglicéridos y aumento del cHDL, en ausencia de modificaciones del CLDL, en tratamiento con fibratos (Rubins 1999).

En los pacientes con diabetes, las concentraciones de cHDL también guardan una relación inversa con la enfermedad cardiovascular (Laakso 1993, Lehto 1997, Howard 2000), y una concentración por debajo de $38 \mathrm{mg} / \mathrm{dl}(1 \mathrm{mmol} / \mathrm{l})$ duplica el riesgo de enfermedad coronaria (Laakso 1993, Lehto 1997). Aunque en los estudios de intervención es difícil separar el efecto del tratamiento sobre el cHDL de otros cambios lipídicos, los datos que existen actualmente sugieren un efecto beneficioso del incremento farmacológico del cHDL (Rubins 1999, Betteridge 2000) (ver más adelante). 


\section{2b-Métodos de medida del colesterol de HDL}

Aunque no existe actualmente un método definitivo de referencia validado para el cHDL, se puede considerar como tal al recomendado por los Centros de Control de Enfermedad (CDC), ya que se sostiene en una sólida base epidemiológica (Hainline 1982). Consiste en la separación de las partícula de VLDL y en la posterior medición del cHDL en el infranadante, tras precipitar las partículas que contienen apoB (heparina/ $\mathrm{Cl}_{2} \mathrm{Mn}$ ) (Hainline 1982). Se suelen considerar igualmente como de referencia a aquéllos métodos que utilizan otros agentes precipitantes como el fosfotungstato$\mathrm{Cl}_{2} \mathrm{Mn}$ o el polietilenglicol. Dado que pocos laboratorios clínicos disponen de una ultracentrífuga, necesaria prara este método de referencia, se considera a la precipitación con sulfato de dextrano en suero/plasma total el método designado de comparación (Wiebe 1997).

En la práctica, aunque la determinación de cHDL puede hacerse por ultracentrifugación o por electroforesis (Wiebe 1997), la mayoría de laboratorios lo hacen por precipitación o por un método de medición directa. El primer método consiste en la precipitación selectiva de las lipoproteinas que contienen apoB (VLDL, LDL, IDL, Ip(a)) mediante la adición de una mezcla de polianiones y cationes divalentes al plasma/suero (heparina/ $\mathrm{Cl}_{2} \mathrm{Mn}$, heparina $/ \mathrm{Cl}_{2} \mathrm{Ca}$, sulfato de dextrano/ $\mathrm{Cl}_{2} \mathrm{Mg}$, fosfotungstato sódico $/ \mathrm{Cl}_{2} \mathrm{Mg}$ o polietilenglicol, generalmente). Posteriormente, se cuantifica el colesterol ( $\mathrm{cHDL}$ ) en el sobrenadante (por un método enzimático) (Wiebe 1997). Más recientemente, se han desarrollado métodos de medición directa del cHDL, sin precipitación, que requieren menor volumen de muestra y son totalmente automatizables. Asimismo, presentan una imprecisión inferior $(<3,1 \%)$, y una inexactitud $(2,2 \%$ vs método recomendado por los CDC) que alcanza los objetivos recomendados por el NCEP (Warnick 1995, Cobbaert 1998, Nauck 1998). El principio es similar a la precipitación, pero los complejos que se forman con las lipoproteinas que contienen apoB son solubles. De esta manera, puede medirse el cHDL sin separar las VLDL y LDL del plasma/suero (Wiebe 1997). 


\section{2c-Colesterol de HDL y diabetes}

Las concentraciones medias de cHDL son inferiores en los hombres y mujeres con diabetes que en los controles del mismo sexo y edad (ver tabla 1a y 1b) (UK Prospective Study Group 1997, Assmann 1988, Siegel 1996, Bangou-Bredent 1999), y la prevalencia de $\mathrm{cHDL}<35 \mathrm{mg} / \mathrm{dl}(<0,9 \mathrm{mmol} / \mathrm{l})$ es del $22-44 \%$ en los hombres con diabetes (Assmann 1988, Siegel 1996), el doble que en la población no diabética de edad similar. El Framingham Offspring Study mostró que un $37,7 \%$ de mujeres con diabetes presentaban $\mathrm{cHDL}<35 \mathrm{mg} / \mathrm{dl}(<0,9 \mathrm{mmol} / \mathrm{l}), 4$ veces la frecuencia de la población control (Siegel 1996), y el Münster Heart Study (PROCAM) mostró una prevalencia similar de mujeres con $\mathrm{cHDL}<45 \mathrm{mg} / \mathrm{dl}(<1,16 \mathrm{mmol} / \mathrm{l})(35,7 \%)$, lo que representa casi el doble que para la población control (Assmann 1998).

\section{3- Triglicéridos}

\section{3a-Trigliceridos y riesgo cardiovascular}

Aunque la relación directa del colesterol total y del cLDL e inversa del cHDL con el riesgo de desarrollar enfermedad cardiovascular está plenamente aceptada, el papel de los triglicéridos como factor de riesgo independiente ha sido más discutido. Aunque Zilversmit en 1979 ya sugería la aterogenicidad de los quilomicrones (Zilversmit 1979) y existen entidades clínicas que cursan con hipertrigliceridemia y que conllevan un riesgo cardiovascular incrementado (Tatami 1981, Steiner 1987, Krauss 1987, Austin 2000), sólo recientemente se ha podido disponer de estudios epidemiológicos que confirmen el papel independiente de los triglicéridos como factor de riesgo cardiovascular (Jeppesen 1998, Assmann 1998). Asimismo, un meta-análisis de 17 estudios prospectivos (Austin 1998) estima que, tras corregir por la concentración de cHDL, por cada $1 \mathrm{mmol} / \mathrm{l}(88,7 \mathrm{mg} / \mathrm{dl})$ que aumenta la concentración plasmática de triglicéridos, existe un incremento del riesgo cardiovascular del 14\% en hombres y del $37 \%$ en mujeres. Por otro lado, y aunque no existen estudios de intervención dirigidos específicamente a pacientes con hipertrigliceridemia, aquéllos en los que existe un 
descenso de los triglicéridos muestran una disminución del riesgo de enfermedad coronaria (Frick 1987, Tenkanen 1994, Rubins 1999), de la mortalidad (Carlson 1988) y de la progresión angiográfica de las estenosis vasculares (Ericsson 1996, Blankenhorn 1987). No obstante, en la mayor parte de estos estudios existen otros cambios lipídicos asociados a la disminución de triglicéridos, como el descenso del cLDL o el aumento del cHDL, que dificultan la interpretación de los resultados.

Otro problema adicional para definir el papel de los triglicéridos en el desarrollo de la aterosclerosis es la gran heterogeneidad de las alteraciones del metabolismo lipoproteico que acompañan a la hipertrigliceridemia, y que las lipoproteinas ricas en triglicéridos (LRT) no son todas igualmente aterogénicas. La progresión de la aterosclerosis coronaria (Phillips 1993, Tornvall 1993, Alaupovic 1997, Hodis 1994) y carotídea (Hodis 1997) es mayor en presencia de concentraciones altas de remanentes de LRT, y la concentración de colesterol de remanentes se asocia a disfunción endotelial coronaria (Kugiyama 1998), infarto de miocardio (Deveraj 1998, Sakata 1998), muerte súbita (Takeichi 1997), reestenosis coronaria tras angioplastia (Tanaka 1995) y nuevos eventos coronarios en pacientes con cardiopatía isquémica previa (Kugiyama 1999).

En la diabetes, la hipertrigliceridemia se ha mostrado como predictor de enfermedad coronaria y mortalidad en numerosos estudios (Fontbone 1989, Laakso 1993, Hanefeld 1996), y el incremento de LRT se relaciona con la gravedad de la ateromatosis coronaria (Tkac 1997). Asimismo, la concentración de triglicéridos preoperatorios predice la supervivencia a largo plazo tras la cirugía de revascularización coronaria (Sprecher 2000).

\section{3b-Métodos de medida de los triglicéridos}

Actualmente, no existe un método estandarizado para la medida de los triglicéridos plasmáticos. Los que más habitualmente se utilizan se basan en la hidrólisis de los triglicéridos (y diglicéridos) y la posterior cuantificación del glicerol liberado de dicha reacción. No obstante, dado que también miden el glicerol endógeno, en situaciones 
que se acompañan de concentraciones altas de glicerol, como la diabetes (Baynes 1991, Puhakainen 1992) pueden darse resultados falsamente incrementados. Para evitar esta interferencia, puede corregirse el resultado final restándole la concentración de glicerol libre existente antes de la hidrólisis (blanco externo) o mediante el consumo del mismo en una reacción previa a la hidrólisis de los triglicéridos (blanco interno) (Cole 1997).

Por otro lado, las lipoproteinas ricas en triglicéridos (LRT) son heterogéneas en cuanto a origen, composición y aterogenicidad, y el aislamiento de determinadas subespecies es difícil (ver más adelante). El desarrollo de un método por cromatografía de inmunoafinidad con un gel anti-apolipoproteina $\mathrm{A} 1 \mathrm{y}$ un anticuerpo monoclonal frente a apoB100 que no reconoce a las partículas ricas en apoE ha permitido separar los remanentes de LRT (los de origen exógeno y endógeno conjuntamente, ver Figura 2) (Nakajima 1993), y los resultados obtenidos con el mismo indican su utilidad para la evaluación del riesgo cardiovascular.

\section{3c-Triglicéridos y diabetes}

Las concentraciones de triglicéridos en los pacientes con diabetes tipo 2 incluidos en los estudios epidemiológicos son invariablemente superiores a las de la población no diabética del mismo sexo y de edad similar, y oscilan entre $180-266 \mathrm{mg} / \mathrm{dl}(2,03-3,0$ $\mathrm{mmol} / \mathrm{l})$ en hombres y entre 140-295 $\mathrm{mg} / \mathrm{dl}(1,58-3,33 \mathrm{mmol} / \mathrm{l})$ en mujeres (Assmann 1988, Barrett-Connor 1982, Siegel 1996, UKPDS27 1997, Haffner 1998) (ver tablas 1a y 1b). Esta diferencia persiste tras ajustar por el índice de masa corporal (BarrettConnors 1982, Siegel 1996), y es también evidente en estudios de intervención con población seleccionada (Koskinen 1992, Pyörälä 1997, Goldberg 1998, Hoofberg 1999) (ver tabla 2).

La prevalencia de la hipertrigliceridemia varía, según el punto de corte utilizado (190$250 \mathrm{mg} / \mathrm{dl}, 2,14-2,82 \mathrm{mmol} / \mathrm{l})$ y la población estudiada, entre $22,6-37,5 \%$ en hombres y entre $21-31 \%$ en mujeres, lo que representa $2-3$ (hombres) y 3-9 veces (mujeres) la frecuencia de la población no diabética (Assmann 1988, Barrett-Connor 1982, Siegel 
1996). No obstante, la mayoría de los pacientes con diabetes tienen concentraciones de triglicéridos sólo leve o moderadamente aumentadas; $1,1-10,6 \%$ de los hombres y $10,9-16,7 \%$ de las mujeres con diabetes presentan concentraciones superiores a 500 mg/l (5,64 mmol/l)(Assmann 1988, Siegel 1996).

Resumen: Las alteraciones lipídicas más frecuentes en la diabetes tipo 2 son la hipertrigliceridemia moderada y el cHDL bajo, alteraciones ambas que se asocian a un aumento del riesgo cardiovascular, y que son susceptibles de ser tratadas. El cLDL es un buen marcador de riesgo en poblaciones amplias, y la adición de los triglicéridos y el cHDL mejora la estimación de dicho riesgo. Cuando se compara con la población no diabética, las mujeres con diabetes presentan perfiles lipídicos relativamente menos favorables que los hombres con diabetes.

\section{COMPONENTES NO CLÁSICOS}

\section{Apolipoproteina B}

\section{1a-Apolipoproteina $\mathrm{B}$ y riesgo cardiovascular}

La apolipoproteina B100 (apoB) forma parte estructural de las partículas de LDL, IDL, VLDL y Ip(a), y permanece unida a ellas desde su síntesis hepática hasta su aclaramiento de la circulación (Berman 1978); sus concentraciones reflejan la masa total de dichas partículas. Dado que cada partícula lipoproteica contiene una sola molécula de apoB, y que el $90 \%$ de ellas está representado por las LDL (Durrington 1978), para una misma concentración de cLDL, un incremento en la apoB sugiere la existencia de partículas con menor razón lípido/proteína, es decir, partículas pequeñas y densas (fenotipo B de las LDL) (Campos 1992).

Las partículas de LDL, tras ser modificadas en la circulación o, especialmente en el espacio subendotelial, se unen a los proteoglicanos de la matriz extracelular a través de una región de la apoB rica en aminoácidos básicos (Camejo 1998, Borén 1998). 
Este papel de la apoB, iniciador del proceso aterogénico, podría explicar su relación con la enfermedad cardiovascular.

Estudios observacionales, tanto transversales como longitudinales, muestran que la apoB es un marcador independiente de riesgo coronario (Durrington 1986, Sigurdson 1992, Tornvall 1993, Lamarche 1996, Westerveld 1998, Graziani 1998) (ver tabla 3). En el estudio cardiovascular de Québec, 2103 varones de edades 45-76 años fueron seguidos durante 5 años. Aquéllos que posteriormente desarrollaron cardiopatía isquémica, mostraban mayor frecuencia de "hiper-apoB" (con cLDL normal) (Lamarche 1995). Las concentraciones de apoB demostraron ser un factor de riesgo coronario independiente (Lamarche 1996, Després 1996), y los fenotipos de dislipemia dependientes de apoB otorgaban un riesgo relativo de enfermedad coronaria comparable al aumento de cLDL (Lamarche 1995). No obstante, no todos los estudios previos ni posteriores al de Québec han confirmado esta asociación independiente entre la apoB y eventos coronarios en prevención primaria (Coleman 1992, Stampfer 1991, Sweetnam 2000). Sin embargo, hay que tener en cuenta que no se ha dispuesto de ensayos de apoB con un estándar internacionalmente aceptado (SP-03) hasta 1996-7, lo que podría en parte explicar las discrepancias entre estudios anteriores y posteriores a esta fecha.

Por otro lado, la reducción de las concentraciones de apoB se asocia con menor progresión de las lesiones coronarias (Brown 1990), especialmente en sujetos con concentraciones de cLDL inferiores a $160 \mathrm{mg} / \mathrm{dl}$ (Stewart BF 1994). Además, la concentración de apoB en sujetos con tratamiento hipolipemiante es mejor predictor de nuevos eventos que el cLDL (van Lennep 2000, Gotto 2000) (Ver tabla 3).

En los sujetos con diabetes, también existe una mayor concentración de apoB en los pacientes con antecedentes de angor inestable, infarto de miocardio, enfermedad cerebrovascular y arteriopatía periférica (Harano 1996). 


\section{1b-Métodos de medida de la apolipoproteina B}

Los métodos utilizados hasta ahora para cuantificar la apoB, basados en el reconocimiento por medio de anticuerpos, son múltiples e incluyen, entre otros, enzimoinmunoanálisis, radioinmunoanálisis, inmunodifusión radial, electroinmunoanálisis y análisis inmunonefelométrico e inmunoturbidimétrico (Bhatnagar 1997). No obstante, la reciente estandarización de los ensayos de apoB con el estándar WHO-IFCC SP-03 ha permitido la comparación teórica entre los resultados de distintos laboratorios (Marcovina 1994), y los resultados procedentes del Framingham Offspring Study han establecido el punto de corte de 1,2 g/l para definir la hiperapoB, por equivalencia a un cLDL de $160 \mathrm{mg} / \mathrm{dl}$ (4,13 mmol/l) (Contois 1996). Sin embargo, a pesar de la estandarización del método, aún existe disparidad de resultados según la población estudiada (Bachorik 1997a, Jungner 1998), probablemente en relación al anticuerpo utilizado. Por tanto, es recomendable referir los resultados a un grupo control normolipémico extraído de la misma población de la que procede el grupo de pacientes a evaluar.

\section{1c-Apolipoproteina B y diabetes}

Los estudios epidemiológicos que incluyen pacientes con diabetes tipo 2 proporcionan escasos datos sobre las concentraciones de apoB en estos sujetos (ver tablas 1a y 1b). El estudio de Framingham muestra concentraciones superiores en mujeres, pero similares en hombres con diabetes que en sujetos del mismo sexo sin diabetes (Siegel 1996). Estudios más recientes revelan concentraciones superiores en los pacientes con diabetes tipo 2 que en los sujetos sin diabetes de similar edad y sexo (BangouBredent 1999) y, entre aquéllos, las concentraciones son aún más altas en los obesos y en los que tienen alguna manifestación macroangiopática (Harano 1996). No obstante, según nuestro conocimiento, no existen datos sobre la prevalencia de hiperapoB en estos pacientes. 


\section{Resumen:}

Los datos existentes sobre apoB en pacientes con diabetes tipo 2 son escasos, pero apuntan a una mayor concentración de esta apolipoproteína en estos sujetos, asociada a su vez a mayor riesgo cardiovascular. Puede ser de utilidad para predecir el riesgo antes de y durante el tratamiento de la dislipemia, su medición está estandarizada y automatizada, y existen valores de poblaciones de referencia.

\section{Partículas de LDL pequeñas y densas}

Las LDL son un conjunto heterogéneo de partículas que difieren en tamaño y densidad, con un rango que oscila entre 1,019 y 1,063 Kg/l. Las más pequeñas y densas (diámetro $\leq 25,5 \mathrm{~nm}$ ) se caracterizan por tener un contenido aumentado en apoproteínas y disminuído en colesterol (razón cLDL/apoB baja)(Campos 1992) y una menor movilidad electroforética (Hokanson 1997). Al predominio éstas partículas pequeñas y densas se le denomina fenotipo B de las LDL (Austin 1988, Austin 1990).

\section{2a-Partículas LDL pequeñas y densas y riesgo cardiovascular}

Entre los múltiples mecanismos que pueden contribuir a una mayor aterogenicidad de las partículas LDL pequeñas y densas que las LDL de mayor tamaño y menor densidad están: su mayor susceptibilidad a la oxidación (Chait 1993, Slyper 1994), su menor afinidad por el receptor de LDL (Galeano 1998), y su mayor capacidad de unión a los proteoglicanos de la pared arterial (Anber 1996) mediante un dominio de la apolipoproteina B100 (Camejo 1998, Boren 1998), que facilita transformaciones ulteriores de la partícula que incrementa su captación por los macrófagos y las células musculares lisas (Heinecke 1998, Hurt-Camejo 1997), también en situación postprandial (Leichleitner 1994). Otros mecanismos implicados incluyen la disfunción endotelial mediada por óxido nítrico (Mäkimattila 1999, Tan 1999) y alteraciones en la fibrinolisis (Festa 1999).

El fenotipo B de las LDL se asocia a un aumento del riesgo coronario, tanto en estudios transversales (Austin 1988, Campos 1992a, Tornvall 1991) como 
longitudinales (Gardner 1996, Stampfer 1996, Lamarche 1996a, Mykkänen 1999). Por otro lado, el tamaño de las partículas de LDL se relaciona de manera inversa con la aterosclerosis carotídea (Skoglund-Andersson 1999, Hulthe 2000) y femoral subclínicas, evaluadas por ultrasonidos (Hulthe 2000), así como con la calcificación coronaria (Superko 1999). No obstante, no todos los estudios sobre la asociación del tamaño de partícula y la enfermedad cardiovascular dan resultados positivos (Sherrard 1996), especialmente en población con concentraciones bajas de cLDL (Gray 1997) y tras ajustar por triglicéridos plasmáticos (Coresh 1993). Aunque ha existido controversia sobre este último punto, un reciente meta-análisis de tres grandes estudios prospectivos muestra un incremento en el riesgo de enfermedad coronaria de 1,6 veces por cada nm que disminuye el diámetro predominante de las partículas. Tras corregir por la concentración de triglicéridos, el riesgo relativo disminuye a 1,3, pero el riesgo continúa siendo significativamente mayor (Austin 1999).

Algunos estudios de intervención muestran que la disminución del colesterol asociado a partículas de LDL densas es el mejor predictor de la progresión angiográfica de aterosclerosis coronaria en hombres con angor pectoris e hipercolesterolemia (Watts 1993) y de la respuesta angiográfica al tratamiento hipolipemiante (Miller 1996), y que la masa de partículas densas también se correlaciona con la progresión angiográfica (Mack 1996).

En pacientes con diabetes, no existen datos epidemiológicos que relacionen el tamaño de las LDL con enfermedad cardiovascular, pero cabría esperar efectos similares que en sujetos no diabéticos. Un pequeño estudio transversal no encontró diferencias en el tamaño predominante de partícula entre sujetos diabéticos con y sin enfermedad coronaria, pero su tamaño (10 paciente por grupo) limita la interpretación de los datos (Tilly-Kiesi 1992).

\section{2b-Medida del tamaño de partícula LDL}

Existen múltiples métodos para caracterizar a las partículas de LDL, que se fundamentan en sus propiedades físicas: flotación, tamaño y densidad. Los más 
utilizados para determinar el predominio de partículas pequeñas y densas son la ultracentrifugación en gradiente de densidad y la electroforesis sobre gel en gradiente de poliacrialamida (Hokanson 1997). El primero, se basa en la preparación de un gradiente discontinuo de sal, que permite separar por ultracentrifugación 3-6 subpoblaciones de LDL según su densidad. En el segundo, se compara la movilidad electroforética de las partículas lipoproteicas con un estándar de diámetro conocido. Ambos métodos son laboriosos y no están al alcance de la mayoría de laboratorios clínicos, y aunque recientemente se ha descrito un método cuantitativo rápido para la medición del tamaño de partícula, éste aún debe ser re-evaluado (Hoefner 2001). Por tanto, sería de utilidad disponer de marcadores del tamaño de partícula, fácilmente cuantificables.

Las concentraciones de triglicéridos explican el $17-50 \%$ de la variabilidad del tamaño de la LDL (Rainwater 2000, Festa 1999, Friedlander 2000, Singh 1995, Gray 1997), y el cHDL explica el 17-39\% de esta variabilidad (Boizel 2000, Singh 1995, Gray 1997,Tchernof 1996). También se han propuesto las razones triglicéridos/cHDL (Boizel 2000) y cLDL/apoB como marcadores de fenotipo B (Campos 1992). La disparidad de resultados de esta última probablemente se deba a los métodos utilizados para determinar el cLDL (Tallis 1995, Furuya 2000), y a la falta de estandarización del ensayo de la apoB hasta recientemente.

\section{2c-Partículas de LDL pequeñas y densas y diabetes mellitus tipo 2}

El tamaño de las LDL es menor en los pacientes con diabetes mellitus tipo 2 que en población no diabética de características similares (Siegel 1996, Mäkimattila 1999). La frecuencia de fenotipo B se encuentra asimismo aumentada, y puede estar presente en hasta $30-50 \%$ de los pacientes con diabetes (Siegel 1996, Caixàs 1997, Abate 1995, Mykkänen 1999, Boizel 2000). Aunque los hombres sin diabetes muestran partículas de LDL más pequeñas y densas que la mujeres (Caixàs 1997, Mc Namara 1987), en pacientes con diabetes no se encuentran diferencias por sexos en la prevalencia de fenotipo B (Caixàs 1997, Haffner 1994). 
Más que la hiperglucemia en sí, parece que son otras alteraciones que frecuentemente la acompañan las que mejor se asocian al tamaño de las LDL (Lahdenperä 1995). Aunque existen varios estudios que muestran una correlación de la resistencia insulínica (Singh 1995, Friedlander 2000, Gray 1997) y el control glucémico (Caixàs 1997) con el tamaño de partícula, ésta correlación no es independiente del resto de componentes que coexisten con la resistencia a la insulina (Singh 1995, Friedlander 2000, Gray 1997). Por otro lado, la mejora del control glucémico se asocia a un descenso en la concentración de triglicéridos y un aumento en el cHDL (Caixàs 1997), parámetros ambos que se correlacionan con el tamaño de las LDL.

Los sujetos con concentraciones de triglicéridos basales superiores a $150 \mathrm{mg} / \mathrm{dl}(1,7$ $\mathrm{mmol} / \mathrm{l}$ ) (Caixàs 1997, Stampfer 1996) (aunque otros autores describen concentraciones inferiores (Griffin 1994, Austin 1990, Syvänne 1996)) y aquéllos con aumento de la lipemia postprandial (Tan 1995, Karpe 1993) tienen mayor predominio de partículas LDL pequeñas y densas. Ambos hechos (aumento de lipemia postprandial y predominio de partículas pequeñas y densas) son patentes en pacientes con nefropatía diabética (Hirano 1998), lo que podría ayudar a justificar el incremento del riesgo cardiovascular en esta población. El incremento postprandial de las lipoproteínas ricas en triglicéridos y la actividad de la lipoproteinlipasa definen en gran parte la heterogeneidad de las partículas LDL en la población no diabética (Karpe 1993). Dado que estos mecanismos son más acusados en la diabetes tipo 2, las partículas LDL pequeñas y densas pueden predominar aun en presencia de buen control metabólico y concentraciones normales de triglicéridos (Abate 1995, Caixàs 1997, Boizel 2000) y de cHDL (Boizel 2000).

\section{Resumen:}

El fenotipo B de las LDL, que se asocia a enfermedad cardiovascular, es una alteración frecuente en la diabetes tipo 2, donde puede estar presente a pesar de un buen control glucémico y un perfil lipídico en ayunas normal. Por tanto, es útil para estimar el riesgo cardiovascular y, si en sujetos con diabetes mantuviera la misma 
relación con el desarrollo de enfermedad cardiovascular que en sujetos sin diabetes, debería incluirse entre los objetivos del tratamiento de la dislipemia en la diabetes tipo 2. Su determinación no es asequible a la mayoría de los laboratorios, por lo que el hallazgo de predictores del fenotipo de las LDL sería de gran utilidad.

\section{Lipemia postprandial}

Tras su absorción intestinal, los triglicéridos de origen exógeno son transportados por la sangre en forma de quilomicrones. Éstos son degradados por la lipoprotein-lipasa endotelial, por la que compiten con las partículas de VLDL (Björkegren 1996, Björkegren 1998); sin embargo, esta enzima muestra mayor afinidad por los quilomicrones, lo que ocasiona un acúmulo postprandial de grandes $\left(\mathrm{VLDL}_{1}\right)$, pero no de VLDL más pequeñas $\left(\mathrm{VLDL}_{2}\right)$ derivadas de las anteriores (Björkegren 1996, Schneeman 1993) (ver Figura 3).

El pico de triglicéridos postprandiales suele presentarse a las 3-4 horas de la ingesta en sujetos normolipémicos, y a las 8 horas (Karpe 1995) de la misma se suele alcanzar la situación basal nuevamente, aunque dependiendo de la magnitud de la ingesta grasa, y de la existencia de diversas alteraciones metabólicas, la lipemia postprandial se puede prolongar más. El $80 \%$ de las partículas ricas en triglicéridos circulantes lo constituyen las VLDL (Schneeman 1993). No obstante, las partículas de quilomicrones y sus remanentes, aunque menor en número, transportan la mayor parte de los triglicéridos postprandiales (Cohn 1993). Por otra parte, el aumento de los triglicéridos postprandiales se acompaña de cambios en la composición de las diferentes partículas lipoproteicas (Björkegren 1998), que determinan su destino metabólico.

\section{3a-Lipemia posprandial y riesgo cardiovascular:}

Tanto los efectos directos de las LRT como las alteraciones lipídicas asociadas a la lipemia postprandial podrían explicar el riesgo cardiovascular asociado al aumento de las mismas. Existen evidencias experimentales de que tras una ingesta rica en grasas 
se produce una disfunción endotelial transitoria (Vogel 1997). Por otro lado, estudios in vitro demuestran que las LRT (tanto VLDL como quilomicrones) promueven la conversión de monocitos en macrófagos, capaces de captar partículas remanentes por mecanismos independientes de receptor apoB/E (Gianturco 1994), y convertirse en células espumosas, protagonistas de la progresión de la placa de ateroma (Gianturco 1994a, Shaikh 1991). Además, se han aislado LRT en las placas de ateroma (Rapp 1994, Mamo 1998). Otro posible mecanismo aterogénico podría estar relacionado con el hecho de que las LRT favorecen un estado procoagulante, mediante la activación del factor VII (Silveira 1994) a través del factor XII activado (Silveira 1996, Mitropoulos 1987) o del factor IX (Miller 1996, Silveira 1996) y la via intrínseca de la coagulación (Mitropoulos 1993). La concentración de VLDL se asocia también positivamente con los niveles de PAI-1, inhibidor de la fibrinolisis (Hamsten 1985), y marcador de riesgo cardiovascular (Cortellaro 1993, Juhan-Vague 1996). Además, el intercambio de colesterol por triglicéridos entre las LRT y las partículas de HDL y LDL, mediado por la proteina transferidora de ésteres de colesterol (PTEC), cuya actividad es triglicéridodependiente (Mann 1991, Tall 1967, Murakami 1995), condiciona cambios aterogénicos en estas partículas. Las LDL, tras enriquecerse en triglicéridos, son más susceptibles de ser hidrolizadas por la lipasa hepática y convertirse en partículas pequeñas y densas (Karpe 1993), más aterogénicas (Austin 1988, Griffin 1994) y son más fácilmente oxidables en situación postprandial en sujetos con diabetes (Diwadkar 1999). Por otro lado, las partículas de HDL enriquecidas en triglicéridos son aclaradas más rápidamente del plasma (Lamarche 1999), lo que disminuye su poder antiaterogénico.

Las concentraciones máximas y tardías de triglicéridos postprandiales, así como las concentraciones de retinil-palmitato, marcadores de LRT de origen exógeno, se han asociado a enfermedad coronaria en multitud de estudios casos-control (Simpson 1990, Groot 1991, Patsch 1992, Gingsberg 1995, Weintraub 1996, Meyer 1996, Karpe 1999), y en un estudio longitudinal (Karpe 1994). Asimismo, las concentraciones de 
triglicéridos postprandiales se asocian con el grado de aterosclerosis carotídea evaluada por ultrasonidos (Karpe 1998, Grønholdt 1996, Sharrett 1995, Boquist 1999, Ryu 1992). No obstante, no existen estudios de intervención que demuestren que la reducción de la lipemia postprandial ocasione una disminución del riesgo de eventos cardiovasculares.

\section{3b-Métodos para la cuantificación de la lipemia postprandial:}

La lipemia postprandial se cuantifica mediante la magnitud del pico o del área bajo la curva de triglicéridos o de LRT de origen endógeno o exógeno, tras una comida de prueba de contenido y composición lipídica conocidos. La determinación de triglicéridos en situación postprandial es indicativa del incremento total de las LRT, tanto de origen endógeno como exógeno, sin diferenciar entre ellas. La medición de ésteres de retinol (en especial el retinil-palmitato) tras una sobrecarga oral de vitamina A (Cortner 1987, Weintraub 1987, Berr 1992) administrada junto con la comida de prueba se utiliza para cuantificar las lipoproteinas ricas en triglicéridos de origen exógeno, es decir los quilomicrones y sus remanentes. Para ello se asumen los siguientes hechos: 1) la incorporación del retinil-palmitato a los quilomicrones secretados por el enterocito (Goodman 1980); 2) que la hidrólisis intravascular (Berr 1984) y la captación extrahepática (Goodman 1980, Bloomhoff 1982) de retinilpalmitato son despreciables; 3) su escasa transferencia a otras lipoproteinas (Cortner 1987, Berr 1984, Wilson 1983); 4) el aclaramiento completo de los quilomicrones del plasma por el hepatocito (Goodman 1980, Bloomhoff 1982); 5) la ausencia de "resecreción" de retinil-palmitato por el hígado (Goodman 1980, Bloomhoff 1982, Berr 1984, Thompson 1983) y 6) la ausencia de niveles plasmáticos significativos de retinilpalmitato en ayunas (Berr 1984). La cuantificación por HPLC de retinil-palmitato en plasma total o en cada una de las fracciones lipoproteicas ha sido la forma más utilizada de determinación de LRT intestinales. Interés similar ha tenido la determinación de $\alpha$-tocoferol tras la ingesta de vitamina $E$ (Demacker 1998). No 
obstante, como se describe más adelante, ambas sustancias presentan algunos inconvenientes como marcadores de los quilomicrones y sus remanentes.

La apolipoproteina B48 es una forma truncada de la apolipoproteina B100, y está constituida el $48 \%$ (extremo aminoterminal) de los aminoácidos de la misma, es sintetizada por el intestino y se mantiene en las LRT de origen intestinal sin aparente intercambio con otras lipoproteinas (Kane 1980, Van't Hooft 1982, Stalenhoef 1984). Sin embargo, la utilización de la apolipoproteina B48 como marcador de lipoproteinas exógenas ha sido obstaculizada por la dificultad de cuantificarla, dado su parecido con la apolipoproteina B100 y su baja concentración plasmática. La electroforesis sobre gel de poliacrilamida ha sido la técnica más utilizada desde que se inició en 1980 (Kane 1980) y fue posteriormente adoptada y modificada por diversos autores (Schneeman 1993, Poapst 1987, Cohn 1988, Zilversmitt 1989, Karpe 1994). La utilización de anticuerpos con afinidad cromatográfica por la apoB100, sin reactividad cruzada por la apolipoproteina B48 ha permitido medir las LRT exógenas mediante HPLC (Deveraj 1998, Nakajima 1996), y la reciente aparición de anticuerpos monoclonales frente a apolipoproteina B48 (Uchida 1998) permitirá la diferenciación de LRT según su origen, intestinal o hepático, por ELISA.

Todos los constituyentes anteriormente detallados son marcadores de la lipemia postprandial. Sin embargo, los resultados que se obtienen con los mismos no son superponibles. Así, el pico de ésteres de retinol suele aparecer retrasado (0-2 horas) respecto al obtenido con la cuantificación de los triglicéridos y de la apolipoproteina B48, y la cinética de esta última es similar a la de los triglicéridos (Karpe 1995). Esto sugiere que existe una producción o aclaramiento distinto de ambos marcadores, como indica algún estudio (Lemieux 1998). Por otro lado, se ha descrito que hasta un $25 \%$ de los esteres de retinol pueden encontrarse en las LRT con apolipoproteina B100 (VLDL o sus remanentes)(Cohn 1993), por transferencia a partir de la tercera hora postprandial (Krasinski 1990, Karpe 1993), que puede alcanzar 19\% en las LDL a las 9 horas y el 32\% a las 12 horas (Cohn 1993, Berr 1984, Wilson 1983, Krasinski 
1990). Este efecto se observa en menor medida también en las HDL (Krasinski 1990). Resultados similares se obtienen cuando se administra vitamina E con la comida de prueba y se mide el $\alpha$-tocoferol en situación postprandial (Demacker 1998). Estos hallazgos ponen en duda la utilidad de las vitaminas liposolubles como marcadores plasmáticos de lipoproteinas de origen exógeno, pero dan información sobre la transferencia lipídica entre quilomicrones y VLDL, y sobre la formación de partículas con un aclaramiento más lento (Demacker 1998).

Independientemente del método utilizado para la medida de la lipemia postprandial, todos ellos coinciden en carecer de valores de normalidad, ya que ni siquiera la comida de prueba ha sido estandarizada. Por tanto, la evaluación de la lipemia postprandial en cualquier grupo de estudio deberá compararse con un grupo control de características similares.

\section{3c-Lipemia postprandial y diabetes}

Las alteraciones lipoproteicas dependientes de triglicéridos, no siempre evidentes en ayunas, se encuentran magnificadas en situación postprandial. Por tanto, el análisis del metabolismo lipídico tras la ingesta de una comida de prueba puede arrojar luz sobre la fisiopatología de la dislipemia diabética, y aclarar algunas de las incógnitas del elevado riesgo cardiovascular del paciente diabético. En la tabla 4 se muestran los principales componentes y mecanismos implicados en la lipemia postprandial en el paciente con diabetes tipo 2 .

La velocidad de la síntesis hepática de las partículas VLDL se encuentra limitada por la disponibilidad de ácidos grasos libres (NEFA) (Frayn 1998, Dixon 1993, Sniderman 1993), que está aumentada en la diabetes mellitus tipo 2, como consecuencia de que el efecto inhibidor de la insulina sobre la lipólisis del tejido adiposo (Chen 1987, Groop 1991) y sobre la síntesis hepática de VLDL1 (Malmstöm 1997) es deficiente. Por otro lado, la síntesis hepática de apolipoproteina B100 está aumentada en la diabetes (Kissebah 1982, Cummings 1995), y la actividad de la lipoprotein-lipasa, dependiente también de insulina, está disminuida en algunos estudios (Deman 1996, Coppack 
1992). Si a la mayor concentración de VLDL inicial y a la menor actividad de la enzima añadimos la aparición de quilomicrones tras la ingesta, que también podría estar aumentada en presencia de determinados polimorfismos (Georgopoulos 2000), cabe esperar una lipemia postprandial incrementada en estos individuos. Sin embargo, los resultados obtenidos en pacientes con diabetes mellitus tipo 2 son controvertidos. En estos sujetos, con frecuencia obesos, los triglicéridos en ayunas ya son superiores a los de la población no diabética. Se ha descrito una mayor área bajo la curva de triglicéridos en pacientes diabéticos, pero la diferencia desaparece al corregir dicha área por los triglicéridos basales (Chen 1993, Syvänne 1994, Cavallero 1994), aunque persisten alteraciones cualitativas en la fracción Sf20-400 (VLDL), con aumento de los remanentes de quilomicrones (Chen 1993a, Syvänne 1994, Attia 1995). Cuando se seleccionan pacientes diabéticos cuyo índice de masa corporal y triglicéridos basales no difieren de los del grupo control, no se encuentran diferencias en el área bajo la curva de triglicéridos en la mayoría de los trabajos (Lewis 1991, Tan 1995, Syvänne 1994a, Durlach 1996, Cooper 1996, Lottenberg 1996, Attia 1995), pero sí en otros (Reznik 1996, Cattin 1996). Por otra parte, se observa una mayor lipemia postprandial, a igualdad de triglicéridos basales, en familiares de primer grado de pacientes con diabetes tipo 2, con insulinresistencia pero con tolerancia normal a la glucosa, que en los controles (Axelsen 1999). Por otra parte, un estudio que comparaba sujetos con intolerancia a la glucosa con sujetos con una sobrecarga oral de glucosa normal y resistencia a la insulina similar (evaluado por el modelo de homeostasis), no mostró triglicéridos y colesterol de remanentes postrandiales superiores en el grupo con intolerancia (Higashi 2000). Estos hallazgos sugieren que la repercusión de la insulinresistencia sobre la tolerancia a los lípidos es más precoz que sobre la de la glucosa, y que es la situación de insulinresistencia en sí, más que la alteración del metabolismo hidrocarbonado, la que está más relacionada con la lipemia postprandial. 
Las LRT son más ricas en apoE en los sujetos diabéticos con enfermedad coronaria que en controles no diabéticos y sin enfermedad coronaria (Syvänne 1994a), lo cual puede justificar una mayor aterogenicidad de las mismas (Chivot 1990), en relación con la mayor captación de las LRT por los macrófagos (Innerarity 1986) y la transformación de éstos en células espumosas. Por otro lado, también se han descrito alteraciones cualitativas, como la glicación de las apolipoproteinas (Curtiss 1985) o su mayor oxidabilidad (Diwadkar 1999), pero la implicación que estos cambios puedan tener sobre la afinidad de las LRT por la lipoprotein-lipasa o el receptor hepático de remanentes no es conocida. Por otra parte, la actividad de la hepático-lipasa está con frecuencia incrementada en la diabetes (Baynes 1991) y en la obesidad (Cominacini 1993), y da como resultado partículas pequeñas y densas, con una baja relación colesterol/proteína (Abate 1995). En la actualidad se asume que la dislipemia diabética es consecuencia de diversas alteraciones, y no existen evidencias que definan a una única de estas múltiples alteraciones enzimáticas y lipoproteicas como desencadenante del resto.

\section{Resumen:}

Las alteraciones lipídicas que caracterizan a la diabetes, moderadas en ayunas, se intensifican en situación postprandial. Aunque la elevada producción hepática de VLDL y la actividad reducida de la lipoproteinlipasa parecen jugar un papel importante en la génesis de la dislipemia diabética, aún faltan estudios que confirmen que aquéllas no son secundarias a otras alteraciones en la lipólisis o en el transporte reverso de colesterol. Tanto la acumulación de LRT como el aumento del número de partículas con predominio de las pequeñas y densas- que caracterizan a estos pacientes, se asocian a enfermedad cardiovascular, y podrían en parte explicar el exceso de riesgo de esta población. 


\section{EVALUACIÓN Y TRATAMIENTO DE LA DISLIPEMIA DIABÉTICA}

Las manifestaciones clínicas de la arteriosclerosis constituyen la principal causa de morbimortalidad en los pacientes con diabetes mellitus, e irán en aumento, a medida que el mejor control de la glucemia reduzca el riesgo de las manifestaciones microvasculares. La diabetes se acompaña, no sólo de mayor riesgo de enfermedad cardiovascular (Coutinho 1999), sino también de peor pronóstico de la misma (Miettinen 1998). Por tanto, el tratamiento, pero sobre todo la prevención de las manifestaciones macrovasculares constituyen uno de los retos más importantes en la atención del paciente con diabetes mellitus.

La diabetes tipo 2 con frecuencia se acompaña de otros factores de riesgo cardiovascular (Assmann 1988). Por tanto, la evaluación clínica del paciente con diabetes debe contemplar el total de los factores de riesgo presentes, y la terapéutica debe ir encaminada a la modificación enérgica de todos ellos (European Diabetes Policy Group 1999, American Association of Clinical Endocrinologists 2000). La dislipemia diabética constituye uno de los factores de riesgo más relevantes, y en la actualidad disponemos de datos que indican que los pacientes con diabetes tipo 2 tienen tanta o más probabilidad que los sujetos no diabéticos de obtener un beneficio con la mejoría de los parámetros lipídicos. 


\section{EVALUACIÓN Y OBJETIVOS TERAPÉUTICOS}

El riesgo de infarto de miocardio fatal en un paciente con diabetes y sin eventos previos es similar al de un paciente sin diabetes con un infarto previo (Haffner 1998), pero los factores de riesgo cardiovascular clásicos no justifican este exceso de riesgo (Stamler 1993). Los organismos internacionales recomiendan la determinación del CLDL, cHDL y triglicéridos en pacientes con diabetes (National Cholesterol Education Progam 1994, American Association of Clinical Endocrinologists 2000, European Diabetes Policy Group 1999). El cLDL constituye el principal factor de riesgo lipídico, tanto en la población diabética como no diabética (ver antes), y la adición del cHDL y los triglicéridos mejora la predicción del riesgo cardiovascular, especialmente en la población diabética (Laakso 1993). Por otra parte, como ya se ha indicado, los pacientes con diabetes presentan otras alteraciones cuantitativas, como el aumento de las lipoproteinas ricas en triglicéridos remanentes (Chen 1993a, Syvänne 1994), y una alta frecuencia de alteraciones lipídicas cualitativas, como el predominio de partículas de LDL y HDL pequeñas y densas, u otras modificaciones de las LDL como la glicación (Curtiss 1985), y el aumento de LDL electronegativas (Sánchez-Quesada 1996). Éstas no se determinan en la valoración de rutina de la dislipemia en la diabetes, pero su evaluación aportaría información adicional sobre el riesgo real de estos pacientes.

Entre los parámetros lipídicos propuestos para evaluar el total de partículas aterogénicas figuran el colesterol no-HDL y la apoB. El primero se calcula restando el cHDL del colesterol total, y refleja el contenido total de colesterol en las partículas aterogénicas, tanto las contabilizadas (LDL, IDL, Lp(a)), como las no contabilizadas habitualmente como LDL, (VLDL y algunos de sus remanentes) (Frost 1998). Por tanto, sería lógico pensar que podría ser especialmete útil en sujetos con hipertrigliceridemia, en los que la correlación entre el cLDL y el colesterol no-HDL es baja. En este sentido, las últimas recomendaciones del National Cholesterol Education Program proponen al colesterol no-HDL como segundo objetivo terapéutico (tras el 
cLDL) en sujetos con diabetes (Expert Panel 2001). Tiene la ventaja de que es sencillo de determinar, y que se relaciona con el riesgo cardiovascular (Frost 1996), e incluso hay datos que sugieren que es mejor predictor de la mortalidad cardiovascular el cLDL (Cui 2001). No obstante, no refleja la masa total de partículas aterogénicas, y no da información sobre la presencia de partículas pequeñas y densas. Por otro lado, aunque no se dispone de datos comparativos entre el colesterol no-HDL y la apoB como predictores de enfermedad cardiovascular, la concentración de apoB sí refleja la masa total de partículas aterogénicas (Berman 1978), su incremento se asocia a enfermedad coronaria (Lamarche 1996), es un buen predictor de riego cardiovascular incluso durante el tratamiento hipolipemiante (Gotto 2000) y su medición está estandarizada (Marcovina 1994). Asimismo, la razón CLDL/apoB puede dar información sobre el tamaño de las partículas de LDL (Campos 1992). En la actualidad no se dispone de marcadores de otras modificaciones cualitativas de la lipoproteinas como la glicación o la oxidación.

En base a la información disponible, y en espera de datos clarificadores sobre la utilidad del colesterol no-HDL y la apoB, el objetivo terapéutico principal, según las recomendaciones internacionales, tanto en pacientes con como sin diabetes, es el cLDL (Expert Panel 1993, Expert Panel 2001, American Diabetes Association 2001), avalado por múltiples estudios de intervención (Sacks 1996, Pyörälä 1997). Dado que el riesgo coronario en los sujetos con diabetes es propio de pacientes en prevención secundaria (Haffner 1998), también se ha adoptado dicho objetivo de tratamiento (cLDL $<100 \mathrm{mg} / \mathrm{dl},<2,6 \mathrm{mmol} / \mathrm{l}$ ) para estos sujetos (American Diabetes Association 2001, Expert Panel 2001). Los objetivos terapéuticos para el cHDL y los triglicéridos, aun siendo alteraciones frecuentes, son más arbitrarios (American Diabetes Association 2001, European Diabetes Policy Group 1999), están menos establecidos y son frecuentemente ignorados. Se consideran deseables niveles de cHDL $>45$ y 55 $\mathrm{mg} / \mathrm{dl}(1,16$ y $1,42 \mathrm{mmol} / \mathrm{l})$ para hombres y mujeres, respectivamente (American Diabetes Association 2001). Para los triglicéridos, aunque el objetivo que 
frecuentemente se propone es $<200 \mathrm{mg} / \mathrm{dl} \quad(<2,25 \mathrm{mmol} / \mathrm{l})$ (American Diabetes Association 2001), concentraciones de triglicéridos superiores a $150 \mathrm{mg} / \mathrm{dl}(1,7 \mathrm{mmol} / \mathrm{l})$ ya se acompañan de alteraciones lipoproteicas aterogénicas como el predominio de partículas LDL pequeñas y densas (Taskinen 1996, Caixàs 1997), y los niveles alcanzados en los ensayos clínicos con estatinas y fibratos están en torno a 130 y 80 mg/dl (1,47 y 0,90 mmol/l), respectivamente (Pyörälä 1997, Sacks 1996, Rubins 1999). Por tanto, parece razonable mantener las concentraciones de triglicéridos por debajo de $150 \mathrm{mg} / \mathrm{dl} \quad(1,7 \mathrm{mmol} / \mathrm{l})$, especialmente en pacientes de riesgo (prevención secundaria o presencia de varios factores de riesgo), tal y como recomiendan las guías europeas (European Diabetes Policy Group 1999), y la última versión de las recomendaciones del NCEP (Expert Panel 2001). Para el colesterol no-HDL, el objetivo recomendado es el del cLDL (según la categoría de riesgo) $+30 \mathrm{mg} / \mathrm{dl}(0,78$ $\mathrm{mmol} / \mathrm{l})$, lo que implica $130 \mathrm{mg} / \mathrm{dl}(3,4 \mathrm{mmol} / \mathrm{l})$ para pacientes con diabetes (Expert Panel 2001) y, según datos de la población de Framingham, el equivalente a los 100 $\mathrm{mg} / \mathrm{dl}(2,6 \mathrm{mmol} / \mathrm{l})$ del cLDL sería aproximadamente $0,8 \mathrm{~g} / \mathrm{l}$ para la apoB (Contois 1996), pero hay que tener en cuenta que este punto de corte puede variar entre distintas poblaciones (Bachorik 1997, Jungner 1998). Aunque existen datos crecientes sobre la utilidad, tanto del colesterol no-HDL como de la apoB en la evaluación del riesgo cardiovascular, los datos de que se dispone aún son insuficientes para hacer recomendaciones concretas, especialmente en los pacientes con diabetes tipo 2. 


\section{TRATAMIENTO}

Dadas las características de la dislipemia diabética, su relación con otras alteraciones presentes en el paciente con diabetes tipo 2, y el efecto de diferentes medidas terapéuticas sobre las diferentes alteraciones asociadas, el esquema de tratamiento más adecuado es la aplicación escalonada de las distintas terapéuticas, empezando por las de mayor espectro de acción sobre los distintos factores de riesgo y continuando con las de efecto más específico sobre las alteraciones lipídicas. El primer escalón incluye, además del abandono del tabaco, el inicio de dieta y ejercicio físico (medidas higiénico-dietéticas); el segundo escalón comprende la optimización del control glucémico con otras medidas y, finalmente, el tercero, la utilización de fármacos hipolipemiantes.

\section{A. Medidas higiénico-dietéticas:}

\section{A1. Dieta y reducción ponderal}

\section{-A1.1: Efectos sobre los componentes clásicos}

Aunque existe controversia sobre el efecto de una dieta rica en ácidos grasos monoinsaturados vs una dieta rica en carbohidratos, las recomendaciones internacionales están de acuerdo en limitar la ingesta de grasas saturadas a menos del $7-10 \%$ de la ingesta calórica total y reducir la ingesta calórica en sujetos con sobrepeso (American Diabetes Association 2000, European Diabetes Policy Group 1999, Expert Pannel on Detection, Evaluation and Treatment of High Blood Cholesterol in Adults 1993). Estas recomendaciones se basan fundamentalmente en los efectos que dichas modificaciones en la dieta tienen sobre el cLDL, el cHDL y los triglicéridos. En hombres obesos sin diabetes, el seguimiento de una dieta isocalórica como la recomendada por la Asociación Americana de Diabetes se asocia a una reducción del $11 \%$ y del $15 \%$ del cLDL y cHDL, respectivamente (Dengel 1995), resultados similares a los obtenidos en sujetos hipercolesterolémicos (Lichtenstein 1994, Flynn 1999). La pérdida de peso con la misma dieta proporciona beneficios adicionales sobre el cLDL 
(Lichtenstein 1994, Flynn 1999, Dengel 1995), ausencia de modificación del cHDL y reducción en las concentraciones de triglicéridos (Dengel 1995, Flynn 1999), que es más acusada cuando la reducción ponderal es mayor (Dengel 1995). Según un metaanálisis de 70 estudios, la pérdida de una media de $16 \mathrm{Kg}$ de peso se acompaña de una reducción del 13\%, $11 \%$ y $32 \%$ en la concentración de colesterol total, cLDL y triglicéridos, respectivamente, y efectos variables sobre el cHDL según se trate de una pérdida de peso en marcha (reducción del 8\%) o ya estabilizada (incremento del 12\%) (Dattilo 1992). Los efectos sobre el perfil lipídico son similares cuando la pérdida ponderal se obtiene mediante la restricción calórica o mediante el ejercicio físico: reducción de triglicéridos y cLDL e incremento en el cHDL (Wood 1988). Sin embargo, la utilización combinada de ambas medidas terapéuticas permite obtener una mayor disminución de peso y mayor efecto sobre los lípidos. En un estudio reciente, controlado y randomizado, que evaluaba el efecto de una dieta baja en grasa y el ejercicio moderado en sujetos sin diabetes mostró una reducción del $7 \%$ en el colesterol total y del $7,5 \%$ en el cLDL en las mujeres, y del $9,1 \%$ en el colesterol total y del $12,8 \%$ en el cLDL en los hombres (la pérdida de peso fue de $5 \mathrm{Kg}$ frente a los controles, mayor que en el grupo tratado con ejercicio solamente) (Stefanick 1998). No obstante, no se observaron cambios significativos tras un año de seguimiento en los grupos a los que se aplicaba una única medida terapéutica (Stefanick 1998).

En los pacientes con diabetes tipo 2 obesos, la reducción intencional de peso (referida por los sujetos de un estudio) disminuyó la mortalidad atribuida a la diabetes y sus complicaciones en un $28 \%$, porcentaje que alcanzó el $33 \%$ en el subgrupo con una reducción entre 10 y $15 \mathrm{Kg}$ (Williamson 2000). Sobre los lípidos, la restricción del consumo energético y la reducción severa de la ingesta de grasa $(6 \%$ del contenido calórico total) disminuyen en un $12,5 \%$ los triglicéridos a los 4 días del inicio de la intervención, tras una pérdida ponderal de $1,6 \%$ del peso inicial. Al mes de iniciar el tratamiento, la reducción de triglicéridos alcanza un $21 \%$, y se observa una reducción también del 22\% en el cLDL (Markovic 1998). No obstante, la composición de este tipo 
de dieta, confeccionada en forma de preparado líquido en el estudio referido (Markovic 1998), es difícilmente alcanzable con alimentación natural. Sin embargo, cambios en el peso obtenidos de forma más gradual con una restricción menor pueden tener un efecto similar sobre el cLDL (Heilbronn 1999). Otros estudios realizados en pacientes con diabetes, que incluyen a un menor número de sujetos, muestran una reducción consistente en la concentración de triglicéridos (Henry 1986, Low 1996, Kelley 1993), y cambios no significativos (Low 1996) o no evaluados (Henry 1986, Kelley 1993) sobre el cLDL y el cHDL (ver tabla 5).

Aunque es difícil diferenciar los efectos independientes de la dieta y de la pérdida ponderal, la reducción de triglicéridos parece depender más de la pérdida de peso, y la reducción del cLDL, de la composición de la dieta (pobre en grasas saturadas) (Heilbronn 1999). Por otro lado, según un reciente meta-análisis, la sustitución de hidratos de carbono por ácidos grasos monoinsaturados produce una reducción neta del $19 \%$ en los triglicéridos y un incremento del $4 \%$ del cHDL, sin efectos significativos sobre el cLDL (Garg 1998).

\section{-A1.2: Efecto de la dieta sobre la apolipoproteína B:}

Sería esperable encontrar una disminución en las concentraciones de apoB en aquellos estudios en los que se objetiva una reducción del cVLDL y del cLDL, pero los trabajos que han valorado el efecto de la dieta sobre esta apolipoproteína son escasos y contradictorios. En un estudio realizado en hombres sanos, una dieta isocalórica, pobre en grasas saturadas redujo las concentraciones de CLDL sin modificar las de apoB en los sujetos con fenotipo $A$, pero sí redujo las concentraciones de apoB en los que tenían fenotipo B de las partículas LDL (Krauss 1995). En otro estudio, realizado en sujetos no obesos con hipercolesterolemia, la restricción de grasa no produjo reducciones en la apoB, a pesar de una disminución en el cLDL. No obstante, cuando fueron sometidos a una dieta hipocalórica, la pérdida de peso de 1,3 $\mathrm{Kg}$ de éstos sujetos sí se acompañó de una reducción en la apoB (Flynn 1999). En un estudio más pequeño, en el que el orden de las dietas no fue randomizado, la restricción en la 
ingesta de grasa ya se acompañó de una reducción en la concentración de apoB, y el efecto se intensificó con la reducción de peso (Lichtenstein 1994). Por el contrario, la comparación, en sujetos obesos normocolesterolémicos, de una dieta baja en grasas y la restricción calórica, no mostró cambios significativos en la apoB, a pesar de una reducción de $10 \mathrm{Kg}$ de peso (Katzel 1995). No obstante, no se comparó la dieta pobre en grasas con la dieta previa de estos pacientes. Finalmente, la reducción ponderal de un $5 \%$ tras un año de tratamiento con dieta y ejercicio se asoció a una disminución significativa en las concentraciones de apoB en los hombres, pero no en las mujeres, a pesar del descenso de cLDL observado en ambos grupos (Stefanick 1998).

En pacientes con diabetes, los datos son aún más escasos. La reducción ponderal de $6 \mathrm{Kg}$ en 28 días, mediante restricción severa del consumo de grasa, en un pequeño grupo de pacientes con diabetes tipo 2 obesos, produjo una disminución significativa de la apoB, lo que indica una reducción en el número de partículas aterogénicas (Markovic 1998).

Por tanto, aunque los resultados son heterogéneos, parece que la reducción calórica y de la ingesta de grasas producen efectos sinérgicos sobre los cambios en las concentraciones de apoB, que dependen, asimismo, de las características de los sujetos y de su perfil lipídico inicial.

\section{A1.3-Dieta y tamaño de las partículas de LDL}

En sujetos sin diabetes, la reducción ponderal por medio de restricción calórica (Katzel 1995, Williams 1990) o aumento de la actividad física (Williams 1990) se asocia a cambios favorables en el tamaño (aumento) y la densidad (disminución) de las partículas de LDL similares con ambas medidas terapeúticas. No obstante, estos cambios favorables no se observan con la disminución de la ingesta grasa sin restricción calórica (Dreon 1998); son dependientes de la pérdida de peso (Williams 1990) y de la reducción en los triglicéridos (Katzel 1995). Asimismo, son más acusados en los sujetos con fenotipo B de las LDL (Katzel 1995, Krauss 1995). 
En pacientes con diabetes tipo 2 obesos y normotrigliceridémicos, una reducción en la ingesta calórica y una disminución severa del consumo de grasa (a un 6\% de la ingesta calórica total) da lugar a un aumento significativo en el tamaño de las partículas LDL, acompañado de una reducción del 12,5\% de triglicéridos y una pérdida ponderal del 1,6\% al 4º día del inicio de la intervención, efecto que se acentúa al mes de tratamiento (Markovic 1998). También se observa un aumento del tamaño de partícula en pacientes con diabetes tipo 2 y obesidad mórbida tras ser sometidos a un by-pass gástrico y perder un $28 \%$ del peso corporal inicial (Barakat 1990).

Por tanto, aunque los efectos de la reducción de grasa aislada son controvertidos, la reducción ponderal incrementa inequívocamente el tamaño de las partículas de LDL, especialmente en aquellos sujetos que inicialmente tienen un fenotipo B de las LDL y concentraciones más altas de triglicéridos.

\section{A1.4-Dieta y lipemia postprandial:}

En sujetos no diabéticos, una dieta rica en grasa saturada incrementa los triglicéridos basales (Weintraub 1988) y el área bajo la curva de los triglicéridos (Weintraub 1988, Harris 1988, Bergeron 1995) y de la apoB48 cuando se compara con una dieta donde predominan los ácidos grasos poli-insaturados, en especial los Ù-3 (Weintraub 1988, Harris 1988). Por otro lado, cuanto mayor es el contenido de grasa de la comida de prueba (Bergeron 1997), mayor es también la lipemia postprandial, siempre que supere los $15 \mathrm{~g}$ (Dubois 1998). Sin embargo, en ausencia de variaciones en la dieta habitual, a igual contenido de grasa, la composición en ácidos grasos de la comida de prueba no influye en el área bajo la curva de triglicéridos (Harris 1988, Thomsen 1999), ésteres de retinol o apolipoproteina B48 (Bergeron 1995, Jackson 1999, Mero 1998).

Los estudios realizados en pacientes con diabetes mellitus tipo 2 son contradictorios. Si bien existen algunos datos que apoyan una mayor lipemia postprandial tras dietas ricas en carbohidratos que tras dietas ricas en ácidos grasos monoinsaturados (Chen 1995) o incluso saturados (Chen 1993), estos resultados no coinciden con la mayoría 
de los estudios, en los que no se encuentran tales diferencias (Campbell 1994, Rasmussen 1993, Rasmussen 1996, Rodríguez-Villar 2000). Por otro lado, en sujetos con diabetes tipo 1, que no tienen insulinresistencia, los escasos datos existentes muestran cambios más favorables tras una dieta rica en hidratos de carbono (Georgopoulos 1998). Esto sugiere que, en pacientes con diabetes tipo 2, la dieta rica en carbohidratos podría aumentar las necesidades de insulina, no corregidas por el tratamiento con hipoglucemiantes orales, y que el empeoramiento del control glucémico explicara la mayor lipemia postprandial de estos sujetos. De hecho, en un estudio donde los pacientes con diabetes tipo 2 recibían tratamiento insulínico (Garg 1988), aquéllos que seguían la dieta rica en carbohidratos requerían mayores dosis de insulina para mantener el control glucémico estable, y aun así persistían glucemias basales más altas. En definitiva, son necesarios más datos antes de poder hacer recomendaciones específicas al respecto.

En cuanto a la composición de ácidos grasos de la dieta, el consumo de ácidos grasos poliinsaturados en pacientes con diabetes condiciona la presencia de mayor número de partículas ricas en triglicéridos postprandiales que si la dieta es rica en monoinsaturados (Madigan 2000).

\section{A2. Ejercicio físico}

\section{A2.1-Efecto sobre los componentes clásicos}

La realización de ejercicio físico regular produce cambios pequeños, pero significativos en los lípidos plasmáticos, como se desprende de un meta-análisis de 31 estudios randomizados: la práctica de ejercicio aeróbico a una intensidad al menos del $50 \%$ del consumo máximo de oxígeno, duración no inferior a 30 minutos por sesión, y frecuencia de tres veces por semana, provoca una reducción de $0,1 \mathrm{mmol} / \mathrm{l}$ en el colesterol total y cLDL y de $0,08 \mathrm{mmol} / \mathrm{l}$ en los triglicéridos y un aumento de 0.05 $\mathrm{mmol} / \mathrm{l}$ en el $\mathrm{cHDL}$ en sujetos normo o hiperlipémicos previamente sedentarios (Halbert 1999). Así mismo, los programas de mayor intensidad tienen mayor efecto 
sobre el cLDL, y los de menor intensidad modifican fundamentalmente el cHDL y los triglicéridos (Halbert 1999).

Los estudios en pacientes con diabetes (ver tabla 6) muestran reducciones de hasta un 33\% en los triglicéridos (Dunstan 1997, Vanninen 1992) y aumentos de hasta el 13\% en el cHDL (Vanninen 1992, Dunstan 1997) cuando se acompañan de reducción ponderal. Sin embargo, en un estudio realizado por nuestro grupo, en el que se mantuvieron constantes el índice de masa corporal y el control glucémico, la práctica de ejercicio físico durante un periodo de tres meses no se acompañó de cambios en las concentraciones de triglicéridos, pero sí de una reducción del $7 \%$ en las concentraciones de cLDL (Rigla 2000). Por el contrario, en un estudio anterior en el que la intensidad del ejercicio y la duración del programa eran menores, no hubo cambios significativos en las concentraciones de cLDL (Dunstan 1997).

Por tanto, el aumento de la actividad física se asocia a una reducción en las concentraciones de triglicéridos, un aumento en el cHDL y efectos más modestos sobre el cLDL, pero que aumentan con la mayor intensidad y duración del ejercicio y cuando éste se acompaña de una pérdida de peso corporal.

\section{A2.2-Ejercicio físico y apolipoproteina B}

Los estudios que valoran el efecto del ejercicio físico sobre las concentraciones de apoB muestran una reducción en las mismas entre un $10 \%$ y un $17 \%$, muy dependiente de la reducción de masa grasa abdominal (Després 1988, Després 1990) y de la intensidad y duración del ejercicio (Lamon-Fava 1989).

Aunque no existen estudios realizados específicamente en sujetos con diabetes, como para el resto de parámetros lipídicos, cabe esperar efectos similares o superiores en estos pacientes, dado que generalmente parten de una situación basal menos favorable que la de los sujetos sin diabetes.

\section{A2.3-Ejercicio físico y tamaño de las partículas de LDL}

Aunque la información es escasa y los sujetos entrenados suelen tener menor proporción de partículas de LDL pequeñas y densas (Williams 1986), sólo la 
realización de un ejercicio tan intenso y duradero como un triatlón (3,8Km de natación, seguido de $180 \mathrm{Km}$ en bicicleta y $42 \mathrm{Km}$ de carrera, duración 12-13 horas) ha conseguido aumentar el tamaño de las partículas de LDL de forma aguda. Además, este efecto únicamente se observó en hombres, y ello asociado a una disminución de los triglicéridos plasmáticos del 67\% y a un aumento en el cHDL del 20\% (Lamon-Fava 1989). La realización de un programa de ejercicio de resistencia de 14 semanas de duración, con un incremento del consumo máximo de oxígeno de un $20 \%$, aunque no modifica el tamaño de las partículas, sí aumenta la razón lípido/proteina por partícula (Houmard 1994). Finalmente, cuando el programa de ejercicio se acompaña de reducción ponderal, se produce un incremento en el tamaño predominante de partícula, de forma similar a lo que sucede con la dieta hipocalórica (Williams 1990).

En pacientes con diabetes, en nuestro conocimiento sólo existe un estudio publicado al respecto, en el que, manteniendo un control metabólico e índice de masa corporal constantes, no se observaron cambios en el tamaño de las partículas de LDL (Rigla 2000).

\section{-Ejercicio físico y lipemia postprandial}

La realización de ejercicio físico antes de una ingesta grasa atenúa la excursión lipídica postprandial (Zhang 1998), aunque la intensidad del ejercicio sea leve (Tsetsonis 1996). Este efecto es independiente de que el ejercicio sea continuo o intermitente (Gill 1998), o lo realicen individuos sedentarios o entrenados, aunque en estos últimos el efecto del ejercicio es más acusado (Tsetsonis 1997). En la población físicamente activa la interrupción del entrenamiento habitual produce un incremento en la lipemia postprandial, efecto ya evidente a las 60 horas de la última sesión de ejercicio, coincidiendo con un incremento significativo de los triglicéridos basales (Hardman 1998). Estos estudios están realizados en población no diabética, pero cabría esperar un efecto tanto o más beneficioso en los sujetos con diabetes tipo 2 y resistencia insulínica. Asimismo, sugieren que el efecto del ejercicio es más agudo que crónico, lo que apoyaría las recomendaciones internacionales de que la práctica de 
ejercicio tenga lugar al menos 3 veces por semana, repartidas uniformemente (American Diabetes Association 2000), con objeto de mantener el efecto de forma permanente.

\section{Resumen:}

Todos los efectos del ejercicio físico sobre los diferentes parámetros lipídicos son favorables y, en general, aditivos a los obtenidos con la dieta y la reducción ponderal. Los efectos más importantes se obtienen sobre los triglicéridos y el cHDL y, aunque los efectos sobre el cLDL son modestos, existe mejoría en la composición de las partículas de LDL.

\section{B. Fármacos hipoglucemiantes: Efectos del control glucémico}

En los pacientes con diabetes tipo 1, la optimización del control glucémico corrige las alteraciones lipídicas hasta normalizarlas (Pérez 2001). En la diabetes tipo 2, sin embargo, los cambios lipídicos que acompañan a la mejora del control, aun siendo favorables, son menos intensos y constantes.

\section{B.1- Efectos sobre los componentes clásicos de la dislipemia diabética.}

El tratamiento intensificado de la hiperglucemia mediante un abordaje escalonado y progresivo de las distintas medidas terapéuticas consigue una reducción en la concentración de triglicéridos a largo plazo (2 años), cuando se compara con un programa estándar de intervención (Emanuele 1998). No obstante, no se observan diferencias entre los cambios inducidos sobre las concentraciones de colesterol total, cHDL y cLDL por ambos programas terapéuticos, a pesar de una diferencia en la HbA1c de 2 puntos (Emanuele 1998).

Aunque todos los agentes orales producen cambios en los lípidos plasmáticos que son dependientes de la mejora en el control glucémico, la metformina ha demostrado disminuir las concentraciones de colesterol total (9\%), cLDL (12\%) y apo(B)(7\%) en sujetos no diabéticos y no obesos (Carlsen 1996), aunque no en obesos (Carlsen 1996, Fontbonne 1996), sin afectar a las concentraciones de glucosa plasmática 
(Carlsen 1996, Fontbonne 1996). En pacientes diabéticos, los estudios en los que se compara la metformina frente al placebo, muestran resultados heterogéneos: la reducción de la HbA1c se sitúa entre 0,5 y 2 puntos (Schneider 1990, Nagi 1993, Robinson 1998, Avilés-Santana 1999), y el efecto sobre los lípidos va desde ausencia de cambios (Avilés-Santana 1999) hasta la reducción del 10-30\% en los triglicéridos (Nagi 1993, Schneider 1990, Lalor 1989) o del 10\% en el cLDL (Nagi 1993, Robinson 1998), sin que coincidan los mayores cambios lipídicos con las mayores reducciones en la HbA1c. Por otra parte, cuando se comparan metformina y sulfonilureas, no se observan diferencias significativas entre las concentraciones lipídicas tras ambos tratamientos (Hermann 1991, Tessier 1999), excepto cuando el tratamiento con metformina se acompaña de mejor control glucémico (De Fronzo 1995). Tampoco la adición de metformina al tratamiento con sulfonilureas produce cambios lipídicos significativos tras tres años de seguimiento en el UKPDS, a pesar del mejor control glucémico con el tratamiento combinado (UK Prospective Study Group 1998).

La adición de tiazolidinas al tratamiento con metformina o insulina ocasiona, a pesar de una mejoría en el control glucémico, un aumento del 8-13\% en el CLDL, acompañado de un incremento del 5-8\% del cHDL, sin modificaciones en los triglicéridos (Schwartz 1998, Buse 1998, Fonseca 2000) ni en la ratio colesterol total/cHDL (Buse 1998, Fonseca 2000). En pacientes sin tratamiento hipoglucemiante previo (Iwamoto 1996) o en tratamiento con sulfonilureas (Iwamoto 1996a, Horton 1998), se observan reducciones de un $13-21 \%$ en los triglicéridos tras el tratamiento con troglitazona, sin cambios significativos en el resto de las lipoproteinas comparado con placebo (Iwamoto 1996a, Horton 1998) (ver tabla 5), posiblemente porque predominen los efectos de mejora del control glucémico.

La optimización del control glucémico mediante tratamiento insulínico, con una reducción de 3-4 puntos en la HbA1c, se acompaña de una reducción del 16-36\% en la concentración de triglicéridos y un incremento del 11-30\% en el cHDL, sin efectos significativos sobre el cLDL (Lindström 1990, Wölffenbuttel 1996, Caixàs 1997, Caixàs 
1997a). Efectos similares se observan con reducciones inferiores de HbA1c, y son dependientes de las concentraciones lipídicas basales de los pacientes estudiados y de si los pacientes experimentan un incremento de peso (Henry 1993, Wolffenbuttel 1993), aunque sólo un estudio ha demostrado reducciones significativas en el cLDL (Wolffenbuttel 1993). Por otro lado, en pacientes previamente insulinizados, el tratamiento con múltiples dosis de insulina subcutánea o bomba de infusión continua intraperitoneal no produce cambios en la concentración de lípidos (Duckworth 1998).

\section{B.2 -Fármacos hipoglucemiantes y apolipoproteina B:}

En un estudio publicado por Emanuele (Emanuele 1998), el tratamiento intensificado de la hiperglucemia mediante un abordaje escalonado y progresivo de las distintas medidas terapéuticas consiguió una reducción en la HbA1c de dos puntos, pero no mostró cambios en las concentraciones de apoB (Emanuele 1998). Por el contrario, existen otros estudios en los que la mejora del control glucémico en tratamiento con insulina o tratamiento combinado sí produce una reducción del $5-11 \%$ en las concentraciones de apoB (Wolffenbuttel 1993, Wolffenbuttel 1996).

\section{B.3-Fármacos hipoglucemiantes y tamaño de las partículas de LDL:}

El tratamiento con troglitazona ha demostrado aumentar el tamaño de partícula en sujetos sin (Sunayama 1999) y con diabetes mellitus (Hirano 1998a), probablemente en relación a los cambios observados en los triglicéridos plasmáticos en estos dos estudios. No obstante, su interpretación ha de ser cautelosa, ya que no se comparó con placebo en ninguno de ellos.

En un estudio realizado por nuestro grupo, la optimización del control glucémico (reducción de 4 puntos de $\mathrm{HbA1c}$ ) con insulina demostró una reducción en la frecuencia de fenotipos no-A de las LDL en pacientes con diabetes mellitus tipo 2, llegando a igualarla a la de los del grupo control (Caixàs 1997). Resultados similares se obtuvieron en un estudio más antiguo que no proporcionó datos sobre la $\mathrm{HbA1c}$ tras el tratamiento (James 1991). Además, a igualdad de control glucémico, el tratamiento con insulina (respecto a las sulfonilureas) se asocia a una reducción en la 
proporción de partículas pequeñas, aun en pacientes normotrigliceridémicos (Rivellese 2000).

El tratamiento intensificado mediante un abordaje escalonado de distintas medidas terapéuticas se asocia a una tendencia a una mayor razón cLDL/apoB, lo que también va a favor de un incremento en el tamaño de las partículas de LDL, que se hacen relativamente más ricas en colesterol y más pobres en triglicéridos (Emanuele 1998).

\section{B.4 -Fármacos hipoglucemiantes y lipemia postprandial:}

La mejoría del control metabólico con glipicida ha demostrado disminuir tanto la trigliceridemia postprandial (mayormente en la fracción de densidad correspondiente a VLDL (Sf 20-400)), como las concentraciones de retinil-palmitato (Jeppesen 1994); este decremento se debe, probablemente, a la disminución de los triglicéridos basales asociados a la reducción de la HbA1c. Resultados similares se han obtenido en pacientes con diabetes tipo 2 tratados con metformina (Jeppesen 1994a). En los pacientes no diabéticos con intolerancia a la glucosa, la metformina no produce modificaciones en los triglicéridos basales ni postprandiales (Grosskopf 1997), pero sí en las concentraciones de retinil-palmitato postprandiales (Grosskopf 1997, Weintraub 1998). Estos datos sugieren que la metformina ejerce un efecto dependiente de la mejora del control glucémico, pero también independiente, disminuyendo la insulinresistencia. El tratamiento con acarbosa, aun en ausencia de cambios en las concentraciones basales, disminuye las excursiones postprandiales tempranas (1h), pero no tardías (5h) de triglicéridos, probablemente en relación a una absorción más lenta de la comida de prueba administrada, rica en carbohidratos (Leonhart 1994).

Los estudios que valoran el efecto del tratamiento insulínico sobre la lipemia postprandial son escasos. Como es de esperar, la disminución de los triglicéridos basales con la mejoría del control metabólico se acompaña de una menor lipemia postprandial, tanto en sujetos con diabetes mellitus tipo 1 como tipo 2 (Georgopoulos 1988). 


\section{Resumen:}

La mejoría del control glucémico, independientemente de las medidas utilizadas, se acompaña de una mejoría del perfil lipídico, que incluye una reducción de la concentración de los triglicéridos basales y postprandiales, un incremento en el cHDL y, en menor medida, una reducción del CLDL y cambios cualitativos, también favorables, en las partículas de LDL.

\section{Fármacos hipolipemiantes:}

\section{D1. Fármacos hipolipemiantes y riesgo cardiovascular.}

La eficacia del tratamiento farmacológico de la dislipemia en los pacientes con diabetes, valorada como la prevención de eventos cardiovasculares, se considera que es similar o superior a la de los pacientes sin diabetes. Sin embargo, el análisis de los estudios de intervención que valoran el efecto de los fármacos hipolipemiantes sobre las manifestaciones cardiovasculares en pacientes con diabetes conlleva una serie de obstáculos: en primer lugar, la mayoría de los estudios no están diseñados para evaluar el efecto del tratamiento hipolipemiante sobre la enfermedad cardiovascular en pacientes con diabetes, y debemos conformarnos con el análisis post hoc de dicho subgrupo (Scandinavian Simvastatin Survival Study Group 1994, Pyörälä 1997, Post Coronary Artery Bypass Graft Trial Investigators 1997, Hoogwerf 1999, Sacks 1996, Goldberg 1998, Rubins 1999); segundo, algunos estudios excluyen expresamente o limitan la inclusión de los pacientes con diabetes (Frick 1997, BIP Study Group 2000); y tercero, la definición de diabetes a menudo no está basada en criterios internacionales (ADA 2000), sino en la necesidad de tratamiento hipoglucemiante (Post Coronary Artery Bypass Graft Trial Investigators 1997, Goldberg 1998). A pesar de estas limitaciones, no hay ningún indicio de que los fármacos hipolipemiantes sean menos eficaces en los pacientes con diabetes y, por el contrario, existen datos consistentes sobre el beneficio de dicho tratamiento en esta población. 
La reducción del cLDL mediante el tratamiento con inhibidores de la HMG-CoAreductasa (estatinas) ha demostrado disminuir el riesgo de eventos cardiovasculares tanto en prevención primaria (Shepherd 1994, Downs 1998) como secundaria (Sacks 1996, Scandinavian Simvastatin Survival Study Group 1994, Long-Term Intervention with Pravastatin in Ischaemic Disease Study Group 1998, Post Coronary Artery Bypass Graft Trial Investigators 1997) en la población general, con efectos equiparables a la angioplastia en pacientes con enfermedad coronaria estable (Pitt 1999). La reducción del riesgo incluye tanto los eventos coronarios (Shepherd 1995, Downs 1998, Sacks 1996, Scandinavian Simvastatin Survival Study Group 1994, Post Coronary Artery Bypass Graft Trial Investigators 1997) como los accidentes cerebrovasculares (Hebert 1997), y se traduce en una reducción en la progresión de la aterosclerosis coronaria en los pacientes sometidos a revascularización (Post Coronary Artery Bypass Graft Trial Investigators 1997). Sin embargo, sólo dos estudios han demostrado una disminución de la mortalidad total (22-30\%) y coronaria (24-42\%) (Scandinavian Simvastatin Survival Study Group 1994, Long Term Intervention with Pravastatin in Ischaemic Disease (LIPID) Study Group 1998), corroborada por un análisis conjunto de 16 estudios randomizados (Hebert 1997).

Los resultados obtenidos del análisis post hoc del subgrupo de pacientes con diabetes sugieren un beneficio relativo similar o superior que el obtenido en los pacientes sin diabetes en la mayoría de los estudios (ver tabla 6). No obstante, solamente el 4 S, un estudio de intervención en pacientes con enfermedad coronaria conocida y concentraciones altas de cLDL (media $188 \mathrm{mg} / \mathrm{dl}, 4,87 \mathrm{mmol} / \mathrm{l}$ ), demostró una reducción de eventos cardiovasculares estadísticamente significativa en dicho subgrupo (Pyörälä 1997). Cuando se incluye en el análisis post hoc a los pacientes con alteración de la glucemia basal (glucosa $>108 \mathrm{mg} / \mathrm{dl} ;>6,0 \mathrm{mmol} / \mathrm{l}$ ), se observa incluso una disminución significativa del $43 \%$ en la mortalidad en este grupo (vs $32 \%$ en los pacientes con glucemia basal normal) (Haffner 1999). A pesar de una reducción similar del riesgo relativo de eventos, dado que la incidencia de enfermedad 
cardiovascular es mayor en los pacientes con diabetes, la reducción absoluta también es mayor en este grupo ( $14 \%$ vs $8 \%$ de eventos a los 6 años). Para prevenir un evento cardiovascular en 5 años en pacientes coronarios sin diabetes hay que tratar con estatinas a 12 sujetos; en pacientes con diabetes es suficiente tratar a 7 (Haffner 1999). Otros estudios que incluyen pacientes con diabetes muestran resultados similares en este subgrupo comparado con los sujetos no diabéticos, pero el número de pacientes es insuficiente para mostrar resultados estadísticamente significativos (Long Term Intervention with Pravastatin in Ischaemic Disease (LIPID) Study Group 1998, Downs 1998).

Los tratamientos farmacológicos cuyo efecto principal es la reducción de triglicéridos y el incremento del cHDL (fibratos) también disminuyen los eventos (Manninen 1988, Rubins 1999) y la mortalidad cardiovasculares (Carlson 1988) en población general, tanto en prevención primaria (Carlson 1988, Manninen 1988) como secundaria (Rubins 1999). Sin embargo, muchos prácticamente excluyen a los pacientes con diabetes (Carlson 1988, Manninen 1988, BIP Study Group 2000).

Los datos de que disponemos en pacientes con diabetes proceden principalmente de estudios de prevención primaria (Manninen 1988, Koskinen 1992, Elkeles 1998). El análisis del pequeño subgrupo de pacientes con diabetes del estudio de Helsinki mostró resultados similares a los observados en el resto de sujetos (ver tabla), pero no consiguió demostrar una reducción estadísticamente significativa de los eventos coronarios (Koskinen 1992). Un reciente estudio, diseñado específicamente en pacientes con diabetes tipo 2, evaluó el efecto de tratar individuos sin enfermedad cardiovascular conocida con fibratos (Elkeles 1998). El seguimiento a 3 años demostró una reducción del $66,7 \%$ en la incidencia de "isquemia probable" en el grupo de tratamiento, pero no consiguió probar un efecto sobre la progresión de la aterosclerosis carotídea o femoral valorada por ultrasonidos, que era el objetivo principal del estudio (Elkeles 1998). El estudio DAIS (Diabetes Atherosclerosis Intervention Study), diseñado para evaluar el efecto de los fibratos sobre la progresión 
de la aterosclerosis coronaria en pacientes con diabetes tipo 2 con enfermedad coronaria angiográfica (Steiner 1996, Steiner 1999), mostró una menor progresión en el grupo de tratamiento, aunque el seguimiento no fue suficiente para mostrar diferencias en la incidencia de eventos cardiovasculares (Diabetes Atherosclerosis Intervention Study Investigators 2001). Los estudios de prevención secundaria proporcionan datos escasos pero prometedores. El tratamiento con fibratos en pacientes con enfermedad coronaria previa y concentraciones normales de cLDL y bajas de cHDL logró una reducción del 22\%, 59\% y 22\% en la incidencia de infarto agudo de miocardio, accidente isquémico transitorio y hospitalización por insuficiencia cardíaca, respectivamente (Rubins 1999). Los resultados obtenidos en el subgrupo de pacientes con diabetes, que constituían el $25 \%$ del total, no difirieron de los resultados generales (ver tabla 6), pero sólo alcanzaron el límite de significación estadística $(p=$ 0,05) (Rubins 1999). Un estudio más reciente, con sólo un $10 \%$ de pacientes con diabetes tipo 2 (la diabetes tipo 1 era criterio de exclusión), y con concentraciones de triglicéridos y cHDL basales similares al anterior sólo, mostró una reducción del infarto o muerte coronaria del $39,5 \%$ en el subgrupo de pacientes con triglicéridos basales superiores a $200 \mathrm{mg} / \mathrm{dl}(2,25 \mathrm{mmol} / \mathrm{l})$, pero no proporcionó información sobre los 310 pacientes con diabetes (BIP Study Group 2000).

Existen estudios de intervención diseñados específicamente para pacientes con diabetes tipo 2, aún en marcha, que posiblemente aporten nuevos datos de utilidad sobre la eficacia del tratamiento de la dislipemia diabética en prevención primaria (CARDS. Fuller JH. Collaborative Atorvastatin Diabetes Study y NIDDM. Non-InsulinDependent Diabetes Mellitus, ambas en: http://www.cardiosource.com/trials; FIELD: Fenofibrate Intervention and Event Lowering in Diabetes. Keech A, comunicación personal; LDS. Lipids in Diabetes Study, con fenofibrato y cerivastatina, Holman RR, comunicación personal) y secundaria (FIELD).

En resumen, fibratos y estatinas parecen tener efectos tanto o más beneficiosos en los pacientes con diabetes que sin ella sobre la reducción del riesgo cardiovascular. 


\section{D2. Fármacos hipolipemiantes y lípidos y lipoproteinas plasmáticas.}

\section{D2.1 -Efectos sobre los componentes clásicos de la dislipemia diabética.}

El efecto del tratamiento hipolipemiante sobre los lípidos plasmáticos en los pacientes con diabetes (ver tablas 6 y 7 ) es similar al que se observa en sujetos sin diabetes. Las estatinas son más eficaces en la reducción del cLDL (Stewart 1994, Jeck 1997, Tikkanen 1998, Goldberg 1990, Gavish 2000), aunque también pueden tener un cierto efecto sobre la reducción de los triglicéridos (Jeck 1997, Tikkanen 1998), dependiente de la concentración de los mismos antes de iniciar el tratamiento y de la potencia hipocolesterolemiante del principio activo (Stein 1998). Los fibratos, por el contrario, tienen mayor efecto sobre los triglicéridos (Stewart 1994, Jeck 1997, Tikkanen 1998, Goldberg 1990, Gavish 2000), con menor efecto sobre el cLDL (Stewart 1994, Jeck 1997, Tikkanen 1998, Goldberg 1990, Gavish 2000, Vega 1990) (ver tabla 7). A pesar de que existen estudios comparativos entre estatinas y fibratos que sugieren un mayor incremento del cHDL con éstos que con aquéllas (Gavish 2000, Tikkanen 1998, Jeck 1997, Niort 1992), esta diferencia no es constante (Goldberg 1990, Vega 1990), y los grandes estudios multicéntricos que incluyen miles de pacientes muestran incrementos de cHDL de 4-7\% tanto con fibratos (Rubins 1999, Elkeles 1998, Koskinen 1992) como con estatinas (Pyörälä 1997, Goldberg 1998, Downs 1998).

Algunos estudios comparativos muestran un incremento del cLDL de hasta un $20 \%$ tras el tratamiento con fibratos (Jeck 1997, Vega 1990, Garg 1989); otros, sin embargo, muestran reducciones en torno a un 10\% (Stewart 1994, Tikkanen 1998), similares a las obtenidas en los grandes estudios multicéntricos (Elkeles 1998, Manninen 1988, Koskinen 1992), en los que la reducción de los triglicéridos está en torno al $10-13 \%$. La diferencia fundamental entre estos estudios radica en las concentraciones basales de triglicéridos. La mayoría de los estudios que muestran triglicéridos basales superiores a 300 mg/dl (Jeck 1997, Garg 1989), aunque no todos (Gavish 2000), demuestran incrementos en el cLDL tras el tratamiento con fibratos. Como explicación, se ha sugerido un mejor catabolismo de las lipoproteinas ricas en 
triglicéridos, que condiciona un cambio en el fenotipo de la partículas de LDL, una transformación en partículas más grandes, más ricas en colesterol, y menos aterogénicas (Jeck 1997) (ver más adelante). Aunque los estudios en pacientes con diabetes son escasos, la combinación de estatinas y fibratos muestra efectos aditivos sobre las lipoproteinas plasmáticas en estos sujetos, sin acompañarse de toxicidad importante (Gavish 2000). Ni fibratos ni estatinas muestran cambios en el control glucémico cuando se comparan con placebo (Stewart 1994) o entre sí (Stewart 1994, Jeck 1997, Goldberg 1990).

Aunque existen menos datos al respecto, también se han estudiado los efectos de otros fármacos en pacientes con diabetes. El ácido nicotínico tiene como efectos principales una reducción de los triglicéridos y el cVLDL, y un incremento del cHDL, pero el empeoramiento del control glucémico en sujetos con diabetes observado en algunos estudios (Garg 1990) ha limitado mucho su uso en estos pacientes. Sin embargo, un estudio reciente a 14 meses sólo muestra una moderada diferencia entre los grupos tratados con ácido nicotínico o placebo (0,3 puntos de HbA1c) (Elam 2000). Su derivado, el acipimox, no ha demostrado efectos deletéreos sobre el control glucémico (Koev 1993, Davoren 1998, Dean 1992, Stewart 1994, Niort 1992) y, aunque se asocia a una reducción del $15-28 \%$ en la concentración de triglicéridos, tampoco provoca un efecto significativo sobre las concentraciones de cHDL cuando se compara con placebo (Koev 1993, Davoren 1998, Dean 1992, Stewart 1994).

Las resinas de intercambio iónico tienen como efecto fundamental la reducción de las concentraciones de cLDL (hasta un $28 \%$ ) en sujetos con diabetes, sin efectos sobre el control glucémico (Garg 1994). Sin embargo, pueden incrementar la concentración de triglicéridos en torno a un 14\% (Garg 1994), por lo que su utilización deberá limitarse al tratamiento de la hipercolesterolemia, en ausencia de hipertrigliceridemia. No existen estudios que exploren los efectos del ácido nicotínico ni de las resinas sobre los eventos cardiovasculares en pacientes con diabetes. 


\section{D2.2 -Efectos de los fármacos hipolipemiantes sobre la apolipoproteina B}

Tanto los fibratos como las estatinas y el acipimox disminyen las concentraciones de apolipoproteina B en pacientes con diabetes (Stewart 1994, Niort 1992, Jeck 1997). No obstante, en estudios comparativos, este efecto es superior con las estatinas, seguidas de los fibratos y el acipimox (Stewart 1994, Niort 1992, Jeck 1997, Frost 2001) (ver tabla 7). Las estatinas reducen la apolipoproteina B asociada a la fracción VLDL+IDL y a la fracción LDL, mientras que los fibratos lo hacen a expensas de la VLDL+IDL (Bredie 1995).

\section{D2.3 -Efectos de los fármacos hipolipemiantes sobre el tamaño de las partículas} de LDL

El tratamiento con fibratos disminuye (frente a placebo, en pequeños estudios de 1012 pacientes) las subpoblaciones más pequeñas de las partículas de LDL (Chapman 1996), y aumenta el tamaño de las partículas (Yuan 1994, Tsai 1992) en pacientes no diabéticos con hipertrigliceridemia. Este efecto se acompaña de modificaciones en la composición de las LDL, que se hacen más pobres en triglicéridos y se enriquecen en ésteres de colesterol (Yuan 1994, Tilly-Kiesi 1991). No obstante, en pacientes normotrigliceridémicos, donde la reducción de los triglicéridos con el tratamiento con fibratos, y la densidad predominante de partícula son menores, no se producen modificaciones en el tamaño de las LDL (Yuan 1994).

El tratamiento con estatinas reduce tanto las partículas de LDL pequeñas como las grandes (Tilly-Kiesi 1991), si bien los datos sobre el tamaño predominante son dispares, y proceden de estudios pequeños, en los que no existe comparación con placebo (Franceschini 1994, Le 2000, Landray 1999, Hoogerbrugge 1999, SánchezQuesada 1999). El único estudio que compara una estatina con placebo no muestra cambios en el tamaño de partícula, aunque tampoco existe una disminución significativa de los triglicéridos (Cheung 1993). Aunque los datos comparativos entre fibratos y estatinas son escasos, demuestran un mayor efecto de los fibratos sobre la densidad predominante de la partículas de LDL, debido a un incremento de las 
fracciones menos densas y una disminución simultánea de las más densas (Bredie 1995). Las estatinas, por el contrario, reducen prácticamente todas las subfracciones, lo que resulta en una disminución del número de partículas, sin mayor cambio en su densidad (Bredie 1995). Otros estudios aportan indicios de resultados similares, como un incremento mayor en la razón $\mathrm{CLDL} / \mathrm{apoB}$ tras el tratamiento con fibratos (Vega 1990).

En pacientes con diabetes, los fibratos producen efectos similares a los descritos en sujetos no diabéticos (Lahdenperä 1993, O'Neal 1998). En un estudio comparativo entre fibratos y estatinas en un pequeño grupo de pacientes con diabetes mellitus tipo 2 y concentraciones moderadamente aumentadas de cLDL (>135 mg/dl, >3,50 mmol/l) y triglicéridos (>200 mg/dl, > 2,25 mmol/l), el tratamiento con fibratos incrementó el tamaño medio de las LDL, mientras que la estatina redujo todas las subpoblaciones de partículas, sin mostrar efecto sobre su patrón de distribución (Frost 2001). Los resultados son similares a los obtenidos en los pacientes con hiperlipemia familiar combinada (Bredie 1995).

El tratamiento con resinas se asocia a una disminución del $20 \%$ en el colesterol asociado a partículas de LDL densas en hombres hipercolesterolémicos con angor pectoris (Watts 1993), pero los datos en sujetos con diabetes son escasos.

\section{D2.4 -Efectos de los fármacos hipolipemiantes sobre la lipemia postprandial}

Los resultados obtenidos con los inhibidores de la hidroxi-metil-glutaril-Co-A reductasa son más heterogéneos que los obtenidos con fibratos, pero en la mayoría de los estudios no disminuyen la lipemia postprandial, ni en sujetos hipercolesterolémicos sin diabetes (Contacos 1998, Cianflone 1990, Weintraub 1989, Cabezas 1993), ni en pacientes con diabetes (Bhatnagar 1995). Estudios más recientes en pacientes sin (Parhofer 2000) y con diabetes tipo 2 (Battula 2000) sí muestran descensos en las concentraciones de triglicéridos postprandiales, asociados a descensos en los triglicéridos basales, pero se trata de estudios sin grupo control (Parhofer 2000), o no randomizados (Battula 2000). 
En sujetos con enfermedad coronaria, el tratamiento con fenofibrato disminuye la trigliceridemia postprandial, asociada a una disminución significativa en los triglicéridos basales (Simpson 1990). Este efecto es similar al producido por otros fibratos en pacientes con hipertrigliceridemia sin (Weintraub 1987, Weintraub 1998, Bhatnagar 1992) o con diabetes (Syvänne 1993, Lewis 1998, Evans 2000), y se acompaña de una mejora tanto basal como postprandial de la respuesta vascular dependiente del endotelio, cuantificada en la arteria braquial mediante ultrasonidos (Evans 2000).

\section{Resumen:}

Todos los fármacos hipolipemiantes son tan efectivos en la reducción de los lípidos plamáticos en los pacientes con como sin diabetes y, por tanto, su selección deberá llevarse a cabo, al igual que en los sujetos no diabéticos, en función de la alteración lipídica predominante. Las estatinas son de elección cuando el cLDL está aumentado y los fibratos, cuando la única alteración es la hipertrigliceridemia. En presencia de dislipemia combinada, dado que el objetivo terapéutico principal es el cLDL, se consideran de elección las estatinas. No obstante, cuando el incremento del cLDL es moderado, el tratamiento óptimo está por definir, especialmente si se tiene en cuenta el efecto favorable de los fibratos sobre el fenotipo de las partículas de LDL. 


\section{JUSTIFICACIÓN Y OBJETIVOS}




\section{JUSTIFICACIÓN}

1. El cLDL es el principal objetivo terapéutico en el manejo de la dislipemia diabética. Sin embargo, la fórmula de Friedewald, el método más utilizado para su determinación, infraestima el valor real del cLDL en los pacientes con diabetes, y el método designado de comparación (la ultracentrifugación) es caro y laborioso, y no está al alcance de la mayoría de los laboratorios clínicos.

2. El riesgo cardiovascular de los pacientes con diabetes no se explica en su totalidad por los factores de riesgo clásicos, incluídos el cLDL y el cHDL. Las alteraciones lipídicas que componen la dislipemia diabética que no se cuantifican rutinariamente (por ejemplo, la lipemia postprandial, la apoB y el fenotipo B de las LDL-predominio de partículas pequeñas y densas) son candidatas potenciales a explicar parte de este riesgo.

-La determinación de la apoB sería de especial utilidad en pacientes con cLDL normal, en los que podría indicar la necesidad y el tipo de tratamiento farmacológico. Se trata de una medición estandarizada y automatizada, asequible a la mayoría de los laboratorios clínicos.

-La medida tanto de la lipemia postprandial como del tamaño de las LDL es laboriosa y requiere equipamientos sofisticados y personal entrenado. Por tanto, sería de utilidad contar con marcadores clínicos o bioquímicos de estas alteraciones, fácilmente asequibles en la práctica clínica habitual, para la evaluación del riesgo global del paciente con diabetes. 
3. En el tratamiento de la dislipemia en la diabetes tipo 2, si no se logran alcanzar los objetivos terapéuticos tras agotar las medidas higiénico-dietéticas y la optimización del control glucémico, deberá iniciarse terapia farmacológica. No obstante, aún está por definir el fármaco o combinación de fármacos más eficaz en el tratamiento de la dislipemia aterogénica que presentan los pacientes con diabetes tipo 2 (hipertrigliceridemia moderada, colesterol HDL bajo y colesterol LDL normal o levemente aumentado, con predominio de partículas de LDL pequeñas y densas). 


\section{OBJETIVOS:}

De acuerdo con las premisas anteriormente enunciadas, los objetivos de la presente tesis han sido:

1. Evaluar métodos alternativos, más exactos que la fórmula de Friedewald, y asequibles a cualquier laboratorio clínico, para la estimación del cLDL en los pacientes con diabetes mellitus tipo 2 .

2. Evaluar los componentes no clásicos de la dislipemia diabética e investigar marcadores clínicos y bioquímicos de los mismos.

a. Evaluar la prevalencia de los fenotipos dislipémicos dependientes del aumento de la concentración de apoB (hiperapoB) en pacientes con diabetes tipo 2 normocolesterolémicos.

b. Evaluar la prevalencia del fenotipo B de las partículas de LDL en sujetos con diabetes tipo 2, y buscar marcadores clínicos y bioquímicos practicables que permitan reconocer su existencia.

c. Comparar la lipemia postprandial de un grupo de pacientes con diabetes tipo 2 con la de un grupo de sujetos no diabéticos, y buscar marcadores (tanto en situación basal como postprandial) de la lipemia postprandial y del tamaño de las partículas de LDL.

3. Comparar la eficacia de un fibrato, una estatina y la combinación de ambos en el tratamiento de los distintos componentes de la dislipemia diabética, especialmente el tamaño de las partículas de LDL. 